\title{
Traces of past activity in the Galactic Centre
}

Gabriele Ponti, Mark R. Morris, Regis Terrier and Andrea Goldwurm

\begin{abstract}
The Milky Way centre hosts a supermassive Black Hole (BH) with a mass of $\sim 4 \times 10^{6} \mathrm{M}_{\odot}$. Sgr $\mathrm{A}^{*}$, its electromagnetic counterpart, currently appears as an extremely weak source with a luminosity $L \sim 10^{-9} \mathrm{~L}_{\text {Edd }}$. The lowest known Eddington ratio $\mathrm{BH}$. However, it was not always so; traces of "glorious" active periods can be found in the surrounding medium. We review here our current view of the Xray emission from the Galactic Center (GC) and its environment, and the expected signatures (e.g. X-ray reflection) of a past flare. We discuss the history of Sgr A*'s past activity and its impact on the surrounding medium. The structure of the Central Molecular Zone (CMZ) has not changed significantly since the last active phase of Sgr $A^{*}$. This relic torus provides us with the opportunity to image the structure of an AGN torus in exquisite detail.
\end{abstract}

\section{The Galactic Centre (GC)}

At approximately $8 \mathrm{kpc}$ (Reid et al. 2009a,b; Matsunaga et al. 2009; Dambis et al. 2009; Gillessen et al. 2009; Vanhollebeke et al. 2009; Trippe et al. 2008; Ghez et

Gabriele Ponti

Max-Planck-Institut für extraterrestrische Physik, Giessenbachstrasse 1, D-85748, Garching bei München, Germany, e-mail:ponti@mpe.mpg.de

Mark Morris

Department of Physics and Astronomy, University of California, Los Angeles 90095-1547, USA

Regis Terrier

Unité mixte de recherche Astroparticule et Cosmologie, 10 rue Alice Domon et Léonie Duquet, 75205 Paris, France

Andrea Goldwurm

Service d'Astrophysique (SAp), IRFU/DSM/CEA-Saclay, 91191 Gif-sur-Yvette Cedex, France Unité mixte de recherche Astroparticule et Cosmologie, 10 rue Alice Domon et Léonie Duquet, 75205 Paris, France 
al. 2008; Groenewegen et al. 2008; Nishiyama et al. 2006; Bica et al. 2006; see Genzel et al. 2010 for a review), the GC is by far the closest Galactic nucleus (e.g. Andromeda, the nearest spiral galaxy, it is $10^{2}$ times further away). Thus it is an excellent laboratory to directly image, with exquisite resolution, the physical processes that might be occurring in many other galactic nuclei.

In the optical window our view toward the Galactic Centre (GC) is obscured by a thick dust curtain, with more than 30 magnitudes of extinction (Becklin \& Neugebauer 1968; Becklin et al. 1978; Rieke et al. 1989), thus preventing any direct view of it. This dust curtain can be raised by observations in different wavebands; a direct view of the GC can be achieved both at longer and shorter wave-lengths, e.g. in infrared-radio as well as medium-hard $(E \geq 2-3 \mathrm{keV}) \mathrm{X}$-rays and $\gamma$-rays. InfraRed (IR) and radio observations have demonstrated that the GC is very bright and display a stupendous variety of phenomena. On large scales ( $\sim 10$ degrees), a large concentration of stars (the so-called Galactic bulge) with a central bar distribution dominates the gravitational potential (de Vaucouleurs 1964; Zhao et al. 1994; Blum 1995). The central few hundred parsecs contain several of the most massive molecular clouds of the Galaxy (Morris \& Serabyn 1996) and radio observations reveal arcs and non thermal filaments (Yusef-Zadeh et al. 1984; Morris 1990; 1992; 1996), indicating the importance of the Galactic magnetic field for the GC environment (Morris \& Serabyn 1996). Within the central few parsecs, IR observations have revealed the presence of a dense and luminous star cluster (Genzel et al. 1996; 2000; 2003; Ghez et al. 2005; 2008; Schodel et al. 2007; 2009; Trippe et al. 2008; Lu et al. 2009). The precise monitoring of stellar motions within $0.5 \operatorname{arcsec}$ of $\mathrm{Sgr}^{*}$. has led to the conclusion that it consists of a compact, dark, central mass concentration of $4 \times 10^{6} \mathrm{M}_{\odot}$, that cannot be anything but a Black Hole (BH, Schodel et al. 2002; Eisenhauer et al. 2003; Ghez et al. 2003; 2005; Gillessen et al. 2009; Genzel et al. 2010).

\subsection{X-ray emission from the $G C$}

The first detections of X-ray sources in the GC date back to the sixties, to the very beginning of X-ray astronomy, when the first short (few minute) observations were made possible with the use of rocket flights (Bowyer et al. 1965; Clark et al. 1965; Fisher et al. 1966; Gursky et al. 1967; Bradt et al. 1968). In the 70s the first soft $\mathrm{X}$-ray satellites (Uhuru, Ariel 5 and HEAO 1) were launched and by the end of the 70s the first GC soft X-ray images, with arcminute resolution, were obtained thanks to the Einstein (HEAO 2) satellite (Watson et al. 1981). Soon after, in the 80s and early 90s, coded-mask imaging techniques allowed a similar revolution in the hard X-ray/soft- $\gamma$-ray spectral regions with the launch of XRT/SpaceLab2 (Skinner et al. 1987), ART-P/GRANAT (Sunyaev et al. 1991; Pavlinsky et al. 1994) and SIGMA/GRANAT (Goldwurm et al. 1994; 2001). These satellites clearly showed that the soft and hard X-ray emission from the GC region is dominated by bright, variable and transient point sources, mainly $\mathrm{X}$-ray binaries. 
Koyama et al. (1989), thanks to GINGA data, were the first to discover (in the GC) a $6.7 \mathrm{keV}$ line (Fe XXV), manifested as diffuse and rather uniform emission, resembling the diffuse Galactic ridge emission (Warwick et al. 1985). Sunyaev et al. (1993), using ART-P data, discovered an additional harder and asymmetric component of the diffuse GC emission. The spatial correlation of this emission with GC Molecular Clouds (MC) prompted the speculation that MC might act as mirrors and this radiation might be reflection of a past flare of a very bright source in the GC (possibly Sgr A* itself). In that case, the authors predicted that the $\mathrm{Fe} \mathrm{K} \alpha$ line, associated with the reflection continuum, should soon be detected. Three years later, owing to the high energy resolution of the ASCA satellite, the hypothesis of a major $\mathrm{X}$-ray flare in the GC was significantly strengthened by the detection of diffuse $\mathrm{Fe}$ $\mathrm{K} \alpha$ line emission from the hard X-ray emitting MC (Koyama et al. 1996). Finally, ASCA's improved energy resolution in the soft X-ray band allowed also the detection of several high-ionisation emission lines (e.g. of $\mathrm{Si}, \mathrm{S}, \mathrm{Ar}, \mathrm{Ca}$, etc.) associated with a low temperature $(\mathrm{T} \sim 0.8-1 \mathrm{keV})$ ionised plasma component pervading the GC.

Since the beginning of this century a new golden age for X-ray astronomy has begun, with the launch of XMM-Newton (1999), Chandra (1999) and Suzaku (2005), three cornerstones in the ESA, NASA and JAXA space projects which have allowed us to take a major step forward in our understanding of the GC X-ray emission.

$\mathrm{X}$-ray observations of the GC show clear evidence for several emission components:

Bright X-ray point sources: For more than 3 decades it has been possible to image the GC X-ray emission (Watson et al. 1981; Churazov et al. 1994; Pavlinsky et al. 1994; Sidoli et al. 2001; Sakano et al. 2002; Sidoli et al. 1999; in't Zand et al. 2004). In particular, during the past decade, intensive Chandra, XMM-Newton, RXTE, INTEGRAL and, on an almost daily basis, Swift monitoring campaigns have been performed within the central 1.2 square degrees (Wang et al. 2002; Baganoff et al. 2003; Muno et al. 2003; 2004; 2006; 2009; Kennea et al. 2006; Degenaar et al. 2009; 2010; 2012; Swank et al. 2001; Kuulkers et al. 2007). Nineteen sources having a 2-10 keV peak luminosity, $\mathrm{L}_{\mathrm{x}} \gtrsim 10^{34} \mathrm{erg} \mathrm{s}^{-1}$, have been detected in this region so far (Degenaar et al. 2012; Wijnands et al. 2006). It has been possible to identify the brightest ones (about half of the sample) as accreting neutron stars or BH. Two of the nineteen sources are persistent (with $\mathrm{L}_{\mathrm{x}} \simeq 10^{35-36} \mathrm{erg} \mathrm{s}^{-1}$ ), while the remaining are transients spending years to decades in a quiescent state with $\mathrm{L}_{\mathrm{x}} \lesssim 10^{33} \mathrm{erg} \mathrm{s}^{-1}$ and undergoing short (weeks/months) outbursts with peak luminosity several orders of magnitudes higher. Ten of the 19 bright sources in the central 1.2 square degrees belong to the class of low-luminosity X-ray transients and have never been observed at luminosity higher than $\mathrm{L}_{\mathrm{x}} \sim 10^{36} \mathrm{erg} \mathrm{s}^{-1}$ and only 3 have reached $\mathrm{L}_{\mathrm{x}} \gtrsim 10^{37}$ erg $\mathrm{s}^{-1}$ (Degenaard et al. 2012; Wijnands et al. 2006). Moreover, no new transients have been found since 2007, suggesting that most X-ray transients recurring on a time scale of less than a decade have now been identified near the GC (in 't Zand et al. 2004; Muno et al. 2009; Degenaar et al. 2010; 2012).

Soft X-ray diffuse plasma: A diffuse soft X-ray emission $\left(\mathrm{E}_{\mathrm{x}} \leqslant 4 \mathrm{keV}\right)$, traced by the H-like and He-like lines of, e.g., Si, S, Ar \& Ca can be best fitted with a low 
temperature $(\mathrm{kT} \sim 0.8-1 \mathrm{keV})$ plasma component that pervades the GC (a small fraction, less than a few percent, of this emission can be ascribed to foreground diffuse soft X-ray emission; Kuntz \& Snowden 2008; Yoshino et al. 2009; Masui et al. 2009; Kimura 2010). This plasma is bound to the Galaxy. Muno et al. (2004), in fact, estimated the sound speed for the soft plasma to be $v_{s} \sim 500 \mathrm{~km} \mathrm{~s}^{-1}$. This plasma will possibly expand to $\sim 1 \mathrm{kpc}$ above the disc, but be bound to the Galaxy (assuming the Breitschwerdt et al. 1991 potential). The plasma radiative cooling time, within the central $50 \mathrm{pc}$, is estimated to be $3-6 \times 10^{6} \mathrm{yr}$ (Muno et al. 2004). If about $1 \%$ of the $10^{51}$ erg of kinetic energy per supernova heats the plasma (as in the Galactic disc; Kaneda et al. 1997), it can be sustained by the reasonable rate of one supernova every $10^{5} \mathrm{yr}$. Additional soft X-ray emission might be produced by interaction of stellar winds from young and massive stars with the interstellar medium (Muno et al. 2004) or each other (Mauerhan et al. 2009) and coronal X-ray sources (Favata et al. 1997; Covino et al. 2000). The patchy soft plasma distribution, with higher concentrations (up to a factor of 10 variations within $50 \mathrm{pc}$ ) towards star forming regions, matches these hypotheses.

Diffuse $6.7 \mathrm{keV}$ (Fe XXV) emission and Galactic ridge X-ray emission: Discovered in the late 70's (Cooke et al. 1970; Bleach et al. 1972; Worral et al. 1982), the Galactic ridge X-ray emission is one of the largest diffuse features (after the X-ray background) in the $2-10 \mathrm{keV}$ band. With a total X-ray luminosity of $\mathrm{L}_{\mathrm{x}}=1-2 \times 10^{38} \mathrm{erg} \mathrm{s}^{-1}$ (Yamauchi et al. 1993; Valinia et al. 1998; Revnivtsev et al. 2006), it is concentrated near the Galactic disc and bulge, extending more than 100 degrees in Galactic longitude and just a few degrees in latitude (Warwick et al. 1985; 1988; Yamauchi et al. 1990; 1993; Revnivtsev 2003). With strong emission lines (e.g. Fe XXV and Fe XXVI at 6.7 and $6.97 \mathrm{keV}$ ) and a hard X-ray continuum, this emission has a spectrum characteristic of a $\mathrm{kT} \sim 5-10 \mathrm{keV}$, optically thin plasma (Koyama et al. 1986; 1989; 2007; Yamauchi et al. 1993; 1996; 2008). However, such a hot plasma cannot be gravitationally or magnetically bound to the Galaxy (Tanaka 2002), thus it would flow away at a supersonic velocity of few thousands $\mathrm{km} \mathrm{s}^{-1}$ (Zel'dovich \& Rayzner 1966), on a timescale of $\sim 3 \times 10^{4}$ years (Sunyaev et al. 1993). To replenish the energy losses a luminosity (supplied throughout the Galaxy) of $10^{43} \mathrm{erg} \mathrm{s}^{-1}$ would be required (Tanaka 2002). At present, no evidence exists for the presence of such a source in the Galaxy.

Alternatively, this diffuse emission might be composed of a large population of initially unresolved, weak point sources (Revnivtsev et al. 2009), as is the extragalactic X-ray background. In support of this hypothesis, it is observed that both the Fe XXV $6.7 \mathrm{keV}$ line and the hard X-ray (3-20 keV) continuum are very well correlated, over the whole Galaxy, with the near IR luminosity $(3-4 \mu \mathrm{m})$ which traces the stellar mass density (Revnivtsev et al. 2006a,b). Moreover, thanks to an ultradeep ( $1 \mathrm{Ms})$ Chandra observation of a field on the Galactic plane $(1=0.08, b=-1.42$, near the GC) with weak interstellar absorption, Revnivtsev et al. (2009) resolved more than $80 \%$ of the diffuse $6-8 \mathrm{keV}$ emission into weak discrete sources such as accreting white dwarfs and coronally active stars. Additionally, the observed luminosity function of these sources is observed to be very similar to the one present in the Solar vicinity (Sazonov et al. 2006; Revnivtsev et al. 2007; 2009). The still un- 
resolved 10-20\% diffuse emission is consistent with being composed of coronally active and normal stars with $\mathrm{L}_{2-10 \mathrm{keV}}<4 \times 10^{29} \mathrm{erg} \mathrm{s}^{-1}$, thus below the detection threshold (Revnivtsev 2009). It now seems clear that the Galactic plane $6.7 \mathrm{keV}$ emission (the so called Galactic ridge diffuse X-ray emission) is mainly composed of weak point sources.

Strong $6.7 \mathrm{keV}$ emission is also present in the GC (Koyama et al. 1989; Yamauchi et al. 1990; Nottingham et al. 1993). As for the Galactic ridge, part of this emission will be produced by accreting white dwarfs and coronally active stars. However, a significant enhancement of $6.7 \mathrm{keV}$ emission, as well as an east-west asymmetry compared to the near IR distributions (tracing point sources), are observed in the central degree (Koyama et al. 2007; 2009; 2011; Yamauchi et al. 2009; Uchiyama et al. 2011). This GC excess of emission (over the best-fit stellar mass distribution model made from near-IR observations and explaining the $6.7 \mathrm{keV}$ emission in the Galactic ridge) is hardly explained by point source origin, requiring a new population of sources with extremely strong Fe XXV line emission (Uchiyama et al. 2011; Tanaka \& Yamauchi 2011). Thus, it has been suggested that this component might be associated with truly diffuse hot gas (Koyama et al. 2007; Uchiyama et al. 2011). The energy required to sustain such hot plasma within the inner $20 \mathrm{pc}$ of the Galaxy is 3 orders of magnitude smaller than for the entire Galaxy, being $\sim 10^{40} \mathrm{erg} \mathrm{s}^{-1}$ (Muno et al. 2004). Belmont et al. (2005) explored the possibility of the hot gas being a gravitationally confined helium plasma. Belmont \& Tagger (2006), instead, suggested that viscous friction of MC flowing within the hot phase can dissipate energy in the gas and heat it. As for the Galactic plane, Chandra can be used to partially resolve the point source contribution to this GC excess of emission. The lower extinction at a distance of 4-9 pc from Sgr A*, allowed Revnivtsev et al. (2007) and Yuasa et al. (2012) to resolve at least $40 \%$ and possibly the bulk of the total 4-8 keV band GC emission into (as in the Galactic ridge) accreting white dwarfs and coronally active stars. The remaining GC $6.7 \mathrm{keV}$ emission might be associated with supernova remnants, such as Sgr A East (Maeda et al. 2002; Sakano et al. 2004; Koyama et al. 2007) which not only show a low temperature (kT $\sim 0.8-1$ $\mathrm{keV})$ plasma component, but also a significantly hotter $(\mathrm{kT} \sim 3-4 \mathrm{keV})$ component producing a harder X-ray continuum and significant Fe XXV line emission.

However, it is difficult to completely rule out that some additional emission might be associated with a hot plasma produced by past energetic events in the GC.

Sgr $\mathbf{A}^{*}$ : One of the major drivers for the deep X-ray surveys of the GC during the past half a century, has been to measure the $\mathrm{X} / \gamma$-ray emission from the supermassive BH associated with the compact radio source Sgr A* (Melia \& Falcke 2001; Melia 2007). A weak source has been detected, at the position of Sgr A*, with a $2-10 \mathrm{keV}$ luminosity of only $\mathrm{L}_{2-10}=2 \times 10^{33} \mathrm{erg} \mathrm{s}^{-1}$ (Baganoff et al. 2003), which corresponds to $4 \times 10^{-12}$ of the Eddington luminosity for a $4 \times 10^{6} \mathrm{M}_{\odot} \mathrm{BH}$. No other BH has ever been detected at such low Eddington ratio. The excellent Chandra spatial resolution allowed also to beautifully resolve the extent of Sgr A*'s quiescent emission to slightly more than 1" (Baganoff et al. 2003), which roughly corresponds to the Bondi radius (Bondi 1952; $r_{\mathrm{Bondi}} \sim 2 \times 10^{5} \mathrm{r}_{\mathrm{g}}$, where $\mathrm{r}_{\mathrm{g}}=G M_{\mathrm{BH}} / c^{2}$ ) and to detect diffuse emission (within 15" radius from Sgr $\mathrm{A}^{*}$ ) from a non-ionisation- 
equilibrium plasma (Xu et al. 2006). Another major result has been the discovery of Sgr A*'s flaring behaviour, both in X-rays (Baganoff et al. 2001; Goldwurm et al. 2003) and near-infrared (Genzel et al. 2003; Ghez et al. 2004). X-ray flares typically occur about once per day, lasting for $\sim 10^{3-4} \mathrm{~s}$, during which the X-ray intensity increases by factors up to 160 (Porquet et al. 2003) from the quiescent value, however the bolometric luminosity still remains extremely low. All these observational properties appear to be consistent with Sgr A* accreting stellar wind material captured from nearby stars (Melia et al. 1992; Coker \& Melia 1997; Rockefeller et al. 2004; Quataert 2004; Cuadra et al. 2005; 2006; 2008).

In the past decade Sgr A* has been repeatedly observed by XMM-Newton and Chandra (as well as in IR), accumulating more than $3 \mathrm{Ms}$ exposure (corresponding to almost two months) and confirming the Sgr A*'s low quiescent-state luminosity. One may wonder whether Sgr A* has always been so underluminous or whether it experienced, in the past, long periods of high-energy activity, that would make the massive black hole of our Galaxy more similar to typical low-luminosity Active Galactic Nuclei, than it appears today.

Diffuse $6.4 \mathrm{keV}(\mathrm{Fe} \mathbf{K} \alpha)$ emission: Extended $\left(\sim 1^{\circ}\right.$, few $\left.10^{2} \mathrm{pc}\right)$, hard $(8-22$ $\mathrm{keV}) \mathrm{X}$-ray emission elongated along the Galactic plane, corresponding spatially with massive Molecular Clouds (MC) was first detected in the early 90's thanks to the ART-P telescope (Sunyaev et al. 1993). The authors interpreted this emission as X-ray reflection of a past energetic event in the GC, predicting the presence of the associated fluorescent $\mathrm{Fe} \mathrm{K} \alpha$ line 1 . Intense, diffuse and patchy $\mathrm{Fe} \mathrm{K} \alpha$ emission, from the same MC, was later measured by ASCA (Koyama et al. 1996), confirming this interpretation. The spatial and temporal variations of the scattered/reflected flux depend on the underlying gas distribution and the past activity of the GC source over a time equal to that taken by the light to cross that region. The presence of massive MC in the inner core of the Galaxy (the so called Central Molecular Zone, CMZ) allows us to put firm constraints on the past activity of the GC over the last $\sim 10^{3} \mathrm{yr}$. This remains true even if other mechanisms can explain the diffuse Fe $\mathrm{K} \alpha$ emission and hard continuum (Valinia et al. 2000; Yusef-Zadeh et al. 2002; 2007; Bykov 2003; Dogiel et al. 2009; 2011).

\section{$2 \mathrm{X}$-ray reflection}

The radiation produced by a powerful X-ray source located close to a MC will interact with the MC material and generate an X-ray reflection component. Hard X-ray

\footnotetext{
${ }^{1}$ Even before the Sgr A* case, the (delayed) reflected emission has been discussed (see Sunyaev et al. 1991; Churazov et al. 1993) for 1E1740.7-2942, one of the two brightest hard X-ray sources in the GC region. During the mid 90s the source, known to be located inside a molecular cloud (Bally \& Leventhal 1991), was observed in a low state with the flux $\sim 5$ times lower than in the bright state. In a set up similar to the one used later for Sgr $\mathrm{A}^{*}$, this fact was used to constrain the optical depth and the size of the cloud from hard X-rays and later from $6.4 \mathrm{keV}$ line (Churazov et al. 1996).
} 

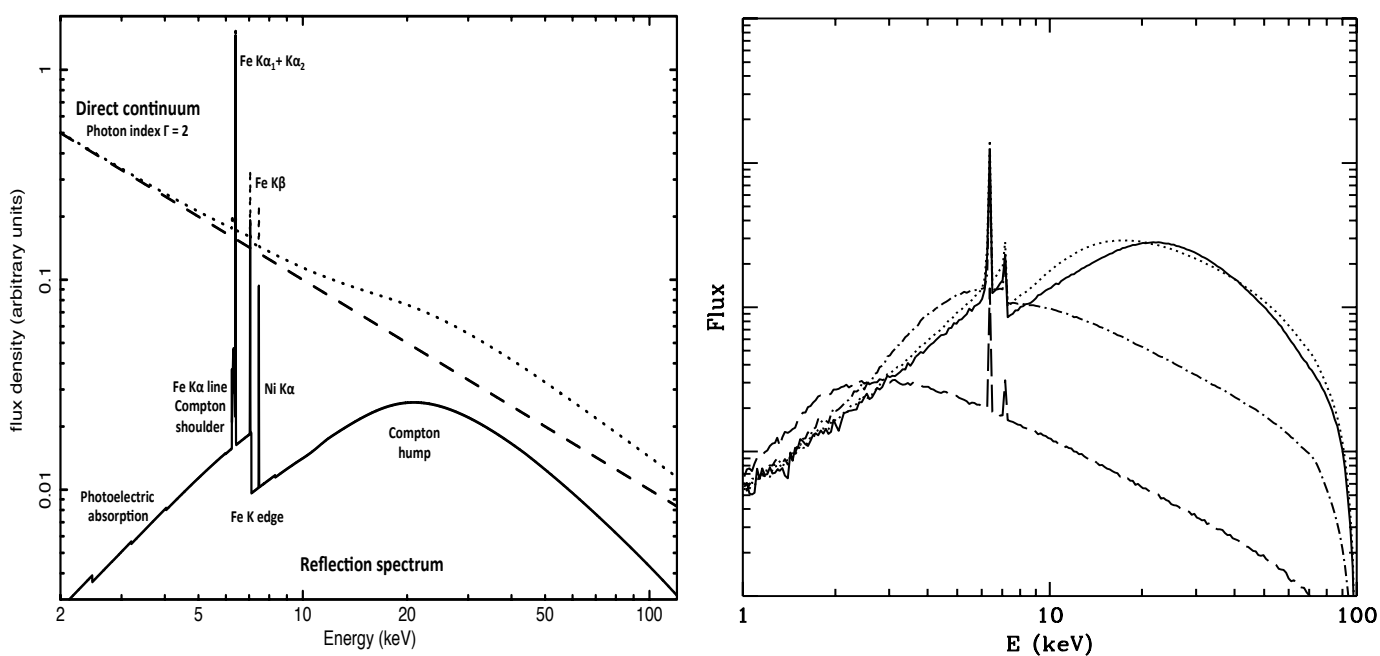

Fig. 1 (Left panel) Typical X-ray reflection spectrum from neutral material with column densities $\sigma_{T} \gg 1$. The $\mathrm{Fe} \mathrm{K} \alpha$ doublet $+\mathrm{K} \beta$ and $\mathrm{Ni} \mathrm{K} \alpha$ lines and the respective Compton shoulders, as well as the $\mathrm{K}$ edges and Compton hump are shown (emission lines from lower atomic number elements which have lower EW are not shown). (Right panel) Reflection spectrum from a molecular cloud (with toroidal geometry and observed face on) for different column densities: $2 \times 10^{22}$ $\mathrm{cm}^{-2}$ (dashed line), $2 \times 10^{23} \mathrm{~cm}^{-2}$ (dot-dashed line), $2 \times 10^{24} \mathrm{~cm}^{-2}$ (dotted line), $2 \times 10^{25} \mathrm{~cm}^{-2}$ (solid line). A prominent Compton hump is expected for $\tau \gg 1$, while the high-energy spectrum changes significantly for smaller column density clouds. Almost no Compton hump is visible for $N_{H}<2 \times 10^{23} \mathrm{~cm}^{-2}$. [Figure courtesy of Matt et al. 2003]

radiation entering a $\mathrm{MC}$ (with typical hydrogen column density of the order of $N_{\mathrm{H}} \sim$ few $10^{22}-10^{24} \mathrm{~cm}^{-2}$; i.e. $\tau \sim 0.01-1$ ) produces, in fact, an X-ray reflection spectrum similar to the one shown in Fig. 1. We describe here the main features of the $\mathrm{X}$-ray reflection from (neutral) MC material. The illuminating source is assumed to have a power law spectrum with photon index $\Gamma=2$.

Continuum: Low energy X-ray radiation is mainly absorbed through the photoionisation of hydrogen, helium and the K-electrons of heavy elements. The photoionisation cross section rapidly decreases with frequency: $\sigma_{\mathrm{ph}} \sim v^{-3}$, thus the Thomson scattering cross section $\left(\sigma_{\mathrm{T}}=6.65 \times 10^{-25} \mathrm{~cm}^{-2}\right)$ already exceeds the photoionisation cross-section per hydrogen atom for photons with energies $\mathrm{E}>$ 8-12 keV (Bethe \& Salpeter 1957; Brown \& Gould 1970; Basko et al. 1974). At energies $\mathrm{E}>$ few $\mathrm{keV}$ the photon wavelength is less than the Bohr radius and electrons bound to hydrogen and helium atoms can be treated as free since the recoil energy $\sim h v\left(h v / m_{e} c^{2}\right)$ greatly exceeds the ionisation potential of these atoms (Basko et al. 1974). High-energy photons lose energy mainly through the recoil effect, transferring it to the electrons at each scattering. The competition between multiple elec- 
tron (down-) scattering of high-energy photons and photoelectric absorption of low energy photons leads to the characteristic "Compton reflection hump" in the reflection spectrum between 20-100 keV (Basko et al. 1974; Fabian 1977; Basko 1978; George \& Fabian 1991; Matt et al. 1991; Nandra \& George 1994; Ghisellini et al. 1994). The left panel of Fig. 1 shows the reflection spectrum from high optical depth $(\tau \gg 1)$ MC. A prominent Compton hump is present between 10 and $50 \mathrm{keV}$. The right panel shows the reflection spectrum from clouds with lower column densities. The reflection spectrum has a very different shape and the Compton hump is less prominent.

Edges and lines: The reflection spectrum does contain jumps corresponding to the K-edges of photo-absorption by heavy elements such as Fe, S, Ar, etc. Due to the high abundance and fluorescent yield of iron, about $30 \%$ of the photons absorbed by photoionisation of iron with energies above the $\mathrm{K}$-shell edge at $7.1 \mathrm{keV}$ give rise to iron $\mathrm{K}$-shell fluorescent photons. The Fe $\mathrm{K} \alpha$ line $(2 \mathrm{p} \rightarrow 1 \mathrm{~s}$ transition) consists of two components $\mathrm{K} \alpha_{1}$ at 6.404 and $\mathrm{K} \alpha_{2}$ at $6.391 \mathrm{keV}$ with branching ratio of 2:1 (Bambynek et al. 1972). The lines have natural widths of $\Delta E \sim 3.5 \mathrm{eV}$. High resolution X-ray spectrometers (such as the one provided by X-ray calorimeters) will resolve the doublet and measure with high accuracy any shift due to the cloud motion. For a MC moving at $100 \mathrm{~km} \mathrm{~s}^{-1}$ a $2.1 \mathrm{eV}$ shift is expected. Since the Mshells of neutral iron atoms are populated, the fluorescent $\mathrm{Fe} \mathrm{K} \beta$ line $(3 \mathrm{p} \rightarrow 1 \mathrm{~s})$ at $7.06 \mathrm{keV}$, with $\mathrm{K} \beta / \mathrm{K} \alpha$ ratio $=0.155-0.16$ (Molendi et al. 2003; Basko 1978; Palmeri $2003 \mathrm{a}, \mathrm{b})$, is also expected.

Because both the $\mathrm{Fe} \mathrm{K} \alpha$ and reflection continuum intensities are roughly proportional to $\tau_{T}$ and source luminosity the Equivalent Width, $\mathrm{EW}_{\mathrm{FeK} \alpha}$ is expected to be $\sim 1 \mathrm{keV}$ ( for Solar abundances), with only $\sim 30 \%$ variations (from $\sim$ 0.7 to $\sim 1 \mathrm{keV}$; see Fig. 3 of Matt et al. 2003) for different MC column densities (Matt et al. 2003). The line EW depends also on the system geometry and the iron abundance $\left(\mathrm{A}_{\mathrm{Fe}}\right)$. For Solar abundances $\left(\mathrm{A}_{\mathrm{Fe}}=1\right)$ the line EW is $\sim 1 \mathrm{keV}$ against the total reflection continuum (Ghisellini et al. 1994; Krolik et al. 1994; Matt et al. 1996; 2003) and it is expected to vary approximately linearly $\mathrm{EW} \sim \mathrm{EW}\left(\mathrm{A}_{\mathrm{Fe}}=1\right) \times \mathrm{A}_{\mathrm{Fe}}^{0.8-0.9}$ for $\mathrm{A}_{\mathrm{Fe}}<<1$ and saturates for $\mathrm{A}_{\mathrm{Fe}}>>1$, $\mathrm{EW} \sim \mathrm{EW}\left(\mathrm{A}_{\mathrm{Fe}}=1\right)\left[1+(0.6-1.2) \times \log \left(\mathrm{A}_{\mathrm{Fe}}\right)\right]$ (Matt et al. 1997). Because of the angular dependence of the Compton effect, the intensity of the scattered continuum (for $\tau \ll 1$ ) measured at an angle $\theta$ with respect to the direction of travel of the incident radiation is expected to be proportional to $\propto\left(1+\cos ^{2}(\theta)\right)$. On the contrary $\mathrm{Fe} \mathrm{K} \alpha$ fluorescence emission is isotropic, thus the observed $\mathrm{EW}_{\mathrm{Fe}}$ has also a dependence on the system geometry. The $\mathrm{K}$-shell fluorescence yield $\left(Y_{Z}^{K}\right)$ is an increasing function of atomic number (Bambynek et al. 1972), thus weak lines are expected from $\mathrm{C}, \mathrm{N}, \mathrm{O}$, and $\mathrm{Ne}\left(Y_{Z}^{K} \sim 10^{-2}\right)$ as well as $\mathrm{Mg}$, Si and $\mathrm{S}\left(Y_{Z}^{K} \sim 10^{-1}\right)$. $\mathrm{EW}_{\mathrm{NiK} \alpha}$ is estimated to be $\sim 0.05 \times \mathrm{EW}_{\mathrm{FeK} \alpha}$ (Yaqoob \& Murphy 2011).

Compton line shoulders: The recoil upon electron scattering shifts the Fe $\mathrm{K} \alpha$ line photons to lower energies (but this is true for any line). Thus, the initial mono-energetic line spreads downward in energy over two Compton wavelengths $\left(\Delta E \simeq 2 E^{2} / m_{e} c^{2}=160 \mathrm{eV}\right.$ ) for each scattering, in this way forming a red wing, the so called "Compton shoulder". The details of the Compton shoulder EW and 
shape depend on the geometry, and the MC column density. For example, for small scattering angles of X-ray photons the recoil energy is comparable or lower than the ionisation energy (Sunyaev \& Churazov 1996), leading to modifications of the shape of the Compton shoulder. The motion of the electrons in hydrogen atoms is another important factor causing strong blurring of the low energy wing of the shoulder (Sunyaev \& Churazov 1996). However, in most cases the Compton shoulder EW is expected to be roughly 10-20 \% of the Fe K $\alpha$ EW (Matt 2002; Yaqoob \& Murphy 2010).

Polarisation: Thomson scattering on electrons will induce linear polarisation of the reflected emission, even in the case of initially unpolarised radiation. The expected degree of polarisation is given by $P=\left(1-\cos ^{2} \theta\right) /\left(1+\cos ^{2} \theta\right)$, where $\theta$ is the scattering angle (Basko et al. 1974; Churazov et al. 2002). The detection of polarised light from the $\mathrm{MC}$ in the GC will provide a clean test of the X-ray external illumination scenario. Moreover, the scattering angle deduced from polarisation (Sunyaev \& Churazov 1998; Odaka et al. 2011) will eventually allow one to determine the MC distance along the line of sight. This is a fundamental parameter for reconstructing the three dimensional gas distributions in the CMZ and the recent history of GC X-ray emission.

Dust scattering halo: The canonical extinction towards the GC is $A_{V} \sim 30 \mathrm{mag}$, which translates to an X-ray absorbing column density of $N_{H} \sim 6 \times 10^{22} \mathrm{~cm}^{-2}$ (Predehl \& Schmitt 1995; Fritz et al. 2011). The interaction of X-rays with interstellar dust grains leads not only to mere absorption, but also to small-angle scattering, producing a faint and diffuse X-ray halo around the source (Overbeck 1965; Martin 1970; Toor et al. 1976; Rolf 1983; Predehl \& Schmitt 1995; Xiang et al. 2007; 2011). Because of the energy dependence of the scattering $\left(I_{\text {halo }} \sim E^{-2}\right)$, dust scattering halos usually appear as a soft power-law component with a fractional intensity of a few to few tens of per cent. The spectral shape of any source in the GC will be modified by the presence of the intervening material. Detailed studies of the dust-scattering halo of bright sources can, in principle, allow one to derive the distribution, along the line of sight, of the scattering material.

\subsection{Time dependent effects}

Depending on the mutual position of the MC and the primary source, substantial evolution of the morphology, flux, equivalent width and shape of the $\mathrm{Fe} \mathrm{K} \alpha$ emission and reflection continuum is expected as a response to a variable primary illuminating source (Sunyaev \& Churazov 1998; Odaka et al. 2011). The light crossing time of the CMZ is $\mathrm{t} \sim 10^{3} \mathrm{yr}$, thus monitoring of the reflection component from

MC provides constrains on the GC X-ray emission on a similar time-scale and also suggests that the observed present-day X-ray reflection component might be associated with a presently dim source that was brighter in the past (Sunyaev et al. 1993; Koyama et al. 1996). 
To a first approximation it is possible to place a direct link between the illuminating source luminosity and the $\mathrm{Fe} \mathrm{K} \alpha$ reflection line flux, assuming isotropic source emission, outside of a cloud with $\tau_{T} \ll 1$ (see e.g. Sunyaev \& Churazov 1998):

$$
F_{\mathrm{FeK} \alpha}=\phi 10^{7} \frac{\Omega}{4 \pi D^{2}} \frac{\delta_{F e}}{3.3 \times 10^{-5}} \tau L_{8} \quad\left(\text { photon } \mathrm{s}^{-1} \mathrm{~cm}^{-2}\right),
$$

where $\phi$ is a factor of order unity which weakly depends on the source spectral shape; $\Omega$ is the solid angle of the cloud subtended from the location of the primary source; $D$ is the distance to the observer; $\delta_{F e}$ is the iron abundance with respect to hydrogen; $\tau$ is the optical depth of the cloud; $L_{8}$ is the continuum source luminosity $\left(\mathrm{erg} \mathrm{s}^{-1}\right.$ ) at $8 \mathrm{keV}$ in the $8 \mathrm{keV}$ wide energy band (see Sunyaev \& Churazov 1998 for definition). Thus: $L_{8}=6 \times 10^{38} \times\left(F_{\mathrm{FeK} \alpha} / 10^{-4}\right) \times\left(0.1 / \tau_{T}\right) \times\left(\delta_{F e} / 3.3 \times\right.$ $\left.10^{-5}\right)^{-1} \times(R / 100 \mathrm{pc})^{2}\left(\mathrm{erg} \mathrm{s}^{-1}\right)$, where $\mathrm{R}$ is the distance from the source to the cloud. If the duration of the source flare is shorter than the cloud light crossing time $\left(\mathrm{r}_{\text {cloud }} / \mathrm{c}\right)$, the luminosity estimates should be multiplied by a factor roughly $\mathrm{r}_{\text {cloud }} / \mathrm{c} \Delta \mathrm{t}$. Because both the Fe $\mathrm{K} \alpha$ and reflection continuum intensities are roughly proportional to $\tau_{T}$ and source luminosity the $\mathrm{EW}_{\mathrm{FeK} \alpha}$ is expected to be $\sim 1 \mathrm{keV}$ (for Solar abundances), with a weak dependence on the MC parameters (Matt et al. 2003).

Light front scanning the CMZ: The right panel of Fig. 3 shows the situation when the CMZ is illuminated by two very short flares from Sgr A*. The light echo seen from a point different from the source is described by an ellipse, i.e. the locus of points so that the sum of the distances to the source and observer is constant. Being the observer at large distance, one focus goes at infinity and the ellipse becomes a parabola. The echo will appear as a parabola, with rotational symmetry about the Z-axis, with equation $z / c=\left(t^{2}-(x / c)^{2}\right) / 2 t$, to an observer at infinity (Churazov \& Sunyaev 1998).

The propagation of an echo in the CMZ allows us to scan the CMZ gas distribution and to perform a tomography of the CMZ. Different patterns of variability are expected for short and long duration flares. In general, the distinction between short and long duration is made on the basis of the light crossing time of the MC and/or MC substructure under investigation. A short duration flare (e.g. months) is expected to produce strong variations of the surface brightness of the $\mathrm{Fe} \mathrm{K} \alpha$ line across the MC image, on angular scales corresponding to the typical size of the MC non-uniformities (down to few arcsec), on the other hand a long duration flare (decades) is expected to reflect the total MC optical depth, which will be much smoother. Monitoring campaigns of the evolution of the Fe K $\alpha$ morphology (in connection with MC column density maps) will allow a disentangling of these different scenarios.

Superluminal propagation of the echo: The apparent echo expansion velocity over the x-coordinate (see Fig. 3) is equal to or larger than the speed of light for any position $\left(|\dot{x} / c|=1+(c t-|x|)^{2} / 2 c t|x|\right)$. Similarly, if the MC is located closer to us than the source, then the echo will scan the MC much quicker than the lightcrossing time of the cloud $\left(\dot{z} / c=0.5+x^{2} / 2(c t)^{2}\right.$, thus $\dot{z}>c$ if $z<0$, when the MC is in front of the source). Superluminal echos can also be observed in particular geo- 
metrical configurations and are mainly linked to the fact that no causal connection is present between the illuminated parts of the MC. The framework is very similar to a projector-screen scenario. The physical variation happens at the level of the projector, for example changing the slide (e.g. taking a time $t_{s}$ ). This variation will appear projected over a distance $d_{\text {wall }}$, thus moving at a velocity $v=d_{\text {wall }} / t_{s}$ which can be $v=d_{\text {wall }} / t_{s} \gg c$ as long as the projector is powerful enough to illuminate a distant enough MC. Observations of superluminal echos have been, and will be, powerful tools to establish the origin of the external illumination of the $\mathrm{Fe} \mathrm{K} \alpha$ emission (Ponti et al. 2010).

Propagation of the $\mathrm{Fe} \mathbf{K} \alpha$ morphology: The morphology and the time evolution of the $\mathrm{Fe} \mathrm{K} \alpha$ emission also provide important constraints, when compared to the MC $N_{H}$ distribution, on the location of the illuminating source (Churazov \& Sunyaev 1998; Murakami et al. 2001; Odaka et al. 2011). A general trend appears where the MC starts to light up from the direction of the source and, if the MC is optically thin $\left(\tau_{T} \ll 1\right)$, the surface brightness distribution reflects the density distribution in the cloud along the surface of the parabola, disappearing on the time-scale of the flare, convolved with the light crossing time of the cloud. In thicker clouds the light front might not penetrate the denser condensations which will then appear as holes (or cast shadows on the more distant parts of the cloud) in the $\mathrm{Fe} \mathrm{K} \alpha$ emissivity if the source is located behind (in front of) the MC (Churazov \& Sunyaev 1998; Murakami et al. 2001; Odaka et al. 2011). The details of the Fe K $\alpha$ morphology thus primarily depend on the MC $N_{H}$ distribution, the duration and light curve of the flare and the source-MC-observer geometry.

Multiple scatterings: Even when the light front has already passed through the MC, multiply scattered photons can still be observed. For example the photons forming Compton shoulders of the lines are created by Thomson scattering of the line photons, thus they will leave the MC with some delay (typically of the order of the light-crossing time of the MC) compared to the primary radiation. Additionally, optically thick and dense MC cores, where multiple scatterings occur, should also still shine for some time after the light front passage. Another effect of multiple scatterings is to dilute the response of the reflection component to fast source variability happening on time-scales shorter than the typical scattering time.

\subsection{X-ray induced chemistry and MC heating}

An X-ray light front irradiating a MC is not only expected to produce an X-ray reflection component, but also to interact with molecules, thus inducing chemical reactions. A correlation between the distribution of $\mathrm{Fe} \mathrm{K} \alpha$ and rotational line emission from the refractory molecule $\mathrm{SiO}$ (with an increase by a factor of $\gtrsim 20$ ) has been observed by Martín-Pintado et al. (2000). This was an indication that either $\mathrm{X}$-rays irradiation or something associated with that is removing the silicon from dust grains in the form of $\mathrm{SiO}$. Amo-Baladrón et al. (2009) confirmed and strengthened this result and suggested that the release of $\mathrm{SiO}$ from small grains can occur 
when hard X-rays deposit their energy in the grains, and heat them locally to high temperature (up to $\sim 10^{3} \mathrm{~K}$ ).

$\mathrm{X}$-ray irradiation might also be the cause of the high temperature of the MC of the CMZ. Rodríguez-Fernández et al. (2004) excluded that X-ray irradiation can be an important source of MC heating. However, they examined present day X-ray luminosities, whereas much brighter past X-ray flares may have occurred at typical intervals much shorter than the cooling time of the $\mathrm{MC}$, so they could be responsible for an important portion of the heating of MC in the GC.

\section{The GC environment}

\subsection{The Central Molecular Zone}

The Central Molecular Zone (CMZ, see Fig. 2) is a thin layer, of about $300 \times 50 \mathrm{pc}$ in size, that contains a total of about $\sim 3-5 \times 10^{7} \mathrm{M}_{\odot}$ of dense $\left(n \sim 10^{4} \mathrm{~cm}^{-3}\right)$, high filling factor $(f \gtrsim 0.1)$ molecular material (Armstrong \& Barrett 1985; Walmsley et al. 1986; Bally et al. 1987; 1988; Gusten 1989; Stark et al. 1989; Tsuboi et al. 1989; Dahmen et al. 1998), which represents $\sim 10 \%$ of the total neutral mass content in the Galaxy (Gusten 1989; Morris \& Serabyn 1996). The MC in the CMZ differ considerably from those in the rest of the Galaxy, with higher gas temperatures $(T \sim 70$ K; Gusten et al. 1981; Morris et al. 1983; Mauersberger et al. 1986), highly supersonic internal velocities $\left(\gtrsim 15-50 \mathrm{~km} \mathrm{~s}^{-1}\right)$ and higher densities $\left(n \sim 10^{4} \mathrm{~cm}^{-3}\right)$. These characteristics are probably deeply related to the special location in the steep gravitational potential of the central bulge, to the GC environment and the past energetic GC activity. For example the high MC densities are probably related to the large tidal shearing present in the CMZ. In fact, as a necessary condition for a cloud at a Galactocentric radius $R_{G}$ to be stable against tidal disruption, its mean density has to be: $n>10^{4} \mathrm{~cm}^{-3} \times\left[75 \mathrm{pc} / R_{G}\right]^{1.8}$ (Gusten \& Downes 1980). Any lower density $\mathrm{MC}$ would be sheared into a tenuous diffuse gas.

The CMZ molecular gas is distributed in giant molecular cloud complexes, with three quarters of the dense clouds located at positive longitudes, three quarters have positive velocities and some have large radial and vertical motions (Bally 1988). This distribution and kinematics are clearly inconsistent with both axial symmetry and uniform circular rotation (Bania 1977; Liszt \& Burton 1978; Morris et al. 1983; Bally et al. 1988; Jackson et al. 1996) and they suggest two different kinematic MC populations.

The disc population: The most massive, lower velocity $\left(v \lesssim 100 \mathrm{~km} \mathrm{~s}^{-1}\right)$ component (the so called "disc population", Bally et al. 1988; Heiligman 1987) resides close to the Galactic plane in the central 100-200 pc and has filament-like clouds with coherent velocity gradients, suggestive of dust lanes and tidally stretched arcs or arms of gas (Stark \& Bania 1986; Serabyn \& Gusten 1987; Bally et al. 1988; Sofue 1995). We point out here that the CMZ disc population appears to have a 


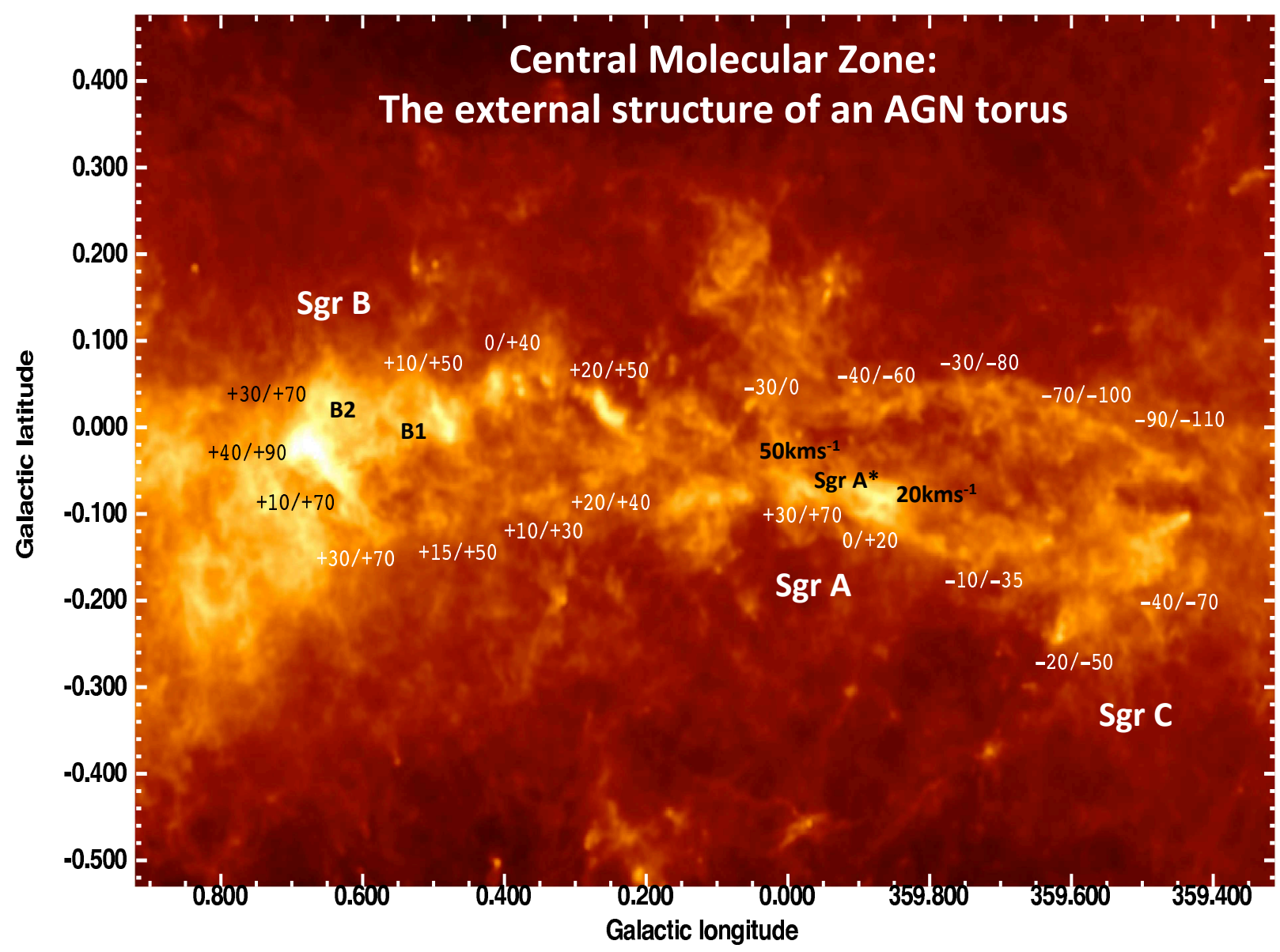

Fig. 2 Herschel map of the atomic hydrogen column density distribution of the central $\sim 150 p c$ of the CMZ showing a large part of the the CMZ disc population. The color scale is logarithmic and extends from $4 \times 10^{22} \mathrm{~cm}^{-2}$ in the darkest regions to $4 \times 10^{25} \mathrm{~cm}^{-2}$ in the brightest MC cores. Velocity information is taken from CS spectroscopic cubes (Tsuboi et al. 1999) for the gas counterparts positionally associated with the dense dust clumps. The CMZ disc population appears to have a geometry and location remarkably similar to the one of the AGN molecular torus invoked by AGN unification schemes (Urry \& Padovani 1995). [Figure courtesy of Molinari et al. 2011]

toroidal geometry (see Fig.2 2 , Molinari et al. 2011) and a physical scale remarkably similar to the outer parts of the AGN molecular torus invoked by AGN unification schemes (Antonucci 1993; Urry \& Padovani 1995). 

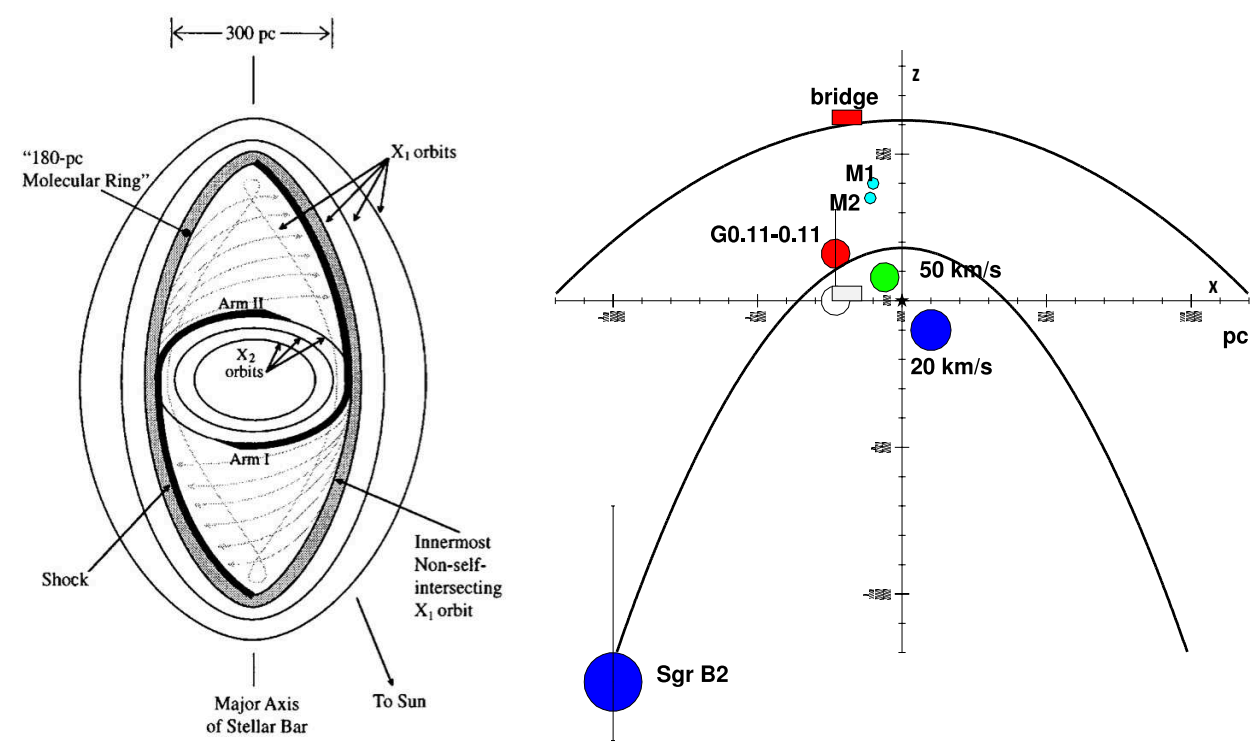

Fig. 3 (Left panel) Schematic diagram illustrating the shapes and relative orientations of the $X_{1}$ and $X_{2}$ orbits and the location of the shocks resulting from the interaction between the innermost $X_{1}$ orbit and the outermost $X_{2}$ orbit. The arms in the CMZ hypothesized by Sofue (1995) are also shown. [Figure courtesy of Morris \& Serabyn 1996]. (Right panel) Sketch of the Galactic plane as seen from the north Galactic pole. Sgr A* is at the vertex and Earth is toward the bottom. Circles represent the hypothesised locations of some MC of the CMZ. Parabolas represent hypothetical light fronts (as seen from Earth) emitted by Sgr A* 100 and 400 yr ago.

180 pc ring: The second, higher velocity $\left(v \gtrsim 130-200 \mathrm{~km} \mathrm{~s}^{-1}\right)$ molecular gas component seems to be located in the outer boundary of the CMZ and it appears to form a quasi-continuous ring structure (the so called " $180 \mathrm{pc}$ ring") inclined by $\sim 20^{\circ}$ with respect to the Galactic plane (Liszt \& Burton 1978; 1980). Further out the $180 \mathrm{pc}$ ring connects with a larger structure with similar tilt, the so called "H I nuclear disc" that contains much of the neutral material $\left(8 \times 10^{6} \mathrm{M}_{\odot}\right.$; Bania 1977; Sofue 1995) lying between 0.3 and $2 \mathrm{kpc}$ from the nucleus (Liszt \& Burton 1978; Gusten 1989; Morris \& Serabyn 1996). Thus the outer part of the $180 \mathrm{pc}$ ring appears to mark a transition between $\mathrm{H}$ I and $\mathrm{H}_{2}$ (Binney et al. 1991).

\section{2 "The Expanding Molecular Ring"}

The H I nuclear ring kinematics are highly perturbed, with outward motions of the same order as the rotation velocities. For this reason it was originally interpreted as a radially Expanding Molecular Ring or EMR (Scoville 1972; Kaifu 1972). Ba- 
nia (1977) proposed that the expansion of this molecular ring was the result of an explosive event at the GC, which occurred $\sim 10^{6} \mathrm{yr}$ ago, providing the momentum impulse to $\sim 10^{7} \mathrm{M}_{\odot}$ of nearby gas. There were two main weaknesses of this interpretation. First is the rather extreme energy release required, being of the order of $E \sim 10^{55} \mathrm{erg}$ (Sanders 1989; Saito 1990), connected with the lack of evidence for the occurrence of such an energetic event. Secondly, the dearth of any clear evidence for interaction between the supposed flood of radially moving molecular material and the non-EMR clouds in the GC region (although Uchida et al. 1994a,b and Sofue 1995 found some indications for shocks as well as gaps in the EMR corresponding to the most prominent clouds in the CMZ). We point out here that the recently discovered Fermi bubbles (Su et al. 2010) would require, if inflated by accretion onto Sgr A*, a similar energy release $E \sim 10^{55} \mathrm{erg}$ occurring on the correct time scale (a few $10^{6} \mathrm{yr}$ ago). Thus part of the kinematical properties of the Expanding Molecular Ring might be carrying the vestige of a period of intense Sgr A* activity.

However, we need to consider that at the distance of few $10^{2} \mathrm{pc}$ from the GC (on the scales of the CMZ and EMR) the gravitational potential is dominated by the stellar component. Nowadays, it is well known that the Galactic bulge has a substantial stellar bar (de Vaucouleurs 1964; Peters 1975; Sellwood 1993; Dwek et al. 1995; Zhao et al. 1994; Blum 1995) extending to at least $2.4 \mathrm{kpc}$ and with a total mass of $1-3 \times 10^{10} \mathrm{M}_{\odot}$. Thus the kinematics of the EMR and CMZ are expected to deviate from axially symmetric circular motion (Binney 1994). In fact, gas moving in response to a bar potential tends to settle into elongated orbits (see the left panel of Fig. 3). When the gas is orbiting at radii between that of corotation and that of the inner Lindblad resonance of the bar pattern, it moves on the so-called $X_{1}$ orbits (see Fig. 3, Contopoulos \& Martzanides 1977), the long axis of which is aligned with the bar. There is an innermost stable $X_{1}$ gas orbit inside of which these orbits become self-intersecting, thus likely orbit-crossing and shocks will happen, leading to angular momentum loss and the gas settling into a new family of gas closed orbits, the $\mathrm{X}_{2}$ orbits, oval-shaped orbits with long axes perpendicular to the bar. In this scenario the $\mathrm{H} \mathrm{I}$ ring would correspond to the $\mathrm{X}_{1}$ orbits, the $180 \mathrm{pc}$ ring being the innermost stable $\mathrm{X}_{1}$ orbit and the inner $\mathrm{CMZ}$ disc population the gas residing on the $X_{2}$ orbits (Binney et al. 1991). A 100 pc elliptical and twisted ring of molecular gas, consistent with residing in $\mathrm{X}_{2}$ orbits, has been recently revealed by new Herschel observations (Molinari et al. 2011), strengthening this interpretation (see Fig. 2).

Probably as a result of the tidal shearing and the associated cloud stability problem (Gusten \& Downes 1980), the CMZ is composed of very clumpy cloud morphology, with about $\sim 10 \%$ of the mass in higher density clumps $\left(\sim 10^{5} \mathrm{~cm}^{-3}\right)$ embedded in a lower density inter-clump medium $\left(\sim 10^{3.5} \mathrm{~cm}^{-3}\right)$. 


\subsection{The CircumNuclear Disc, CND}

The inner $5 \mathrm{pc}$ from $\mathrm{Sgr} \mathrm{A}^{*}$ contains a concentration (with total mass of $\sim 10^{5-6}$ $\mathrm{M}_{\odot}$ ) of dense and warm molecular and atomic gas (the so called CircumNuclear Disc, CND, Becklin et al. 1982; Genzel et al. 1985; Gusten et al. 1987; Christopher et al. 2005; Montero-Castano et al. 2009; Oka et al. 2011; Martin et al. 2012; Requena-Torres et al. 2012) with a configuration similar to a rotating disc and/or a set of filaments with a tilt of $20-30^{\circ}$ compared to the Galactic plane and very dense $\left(n_{H} \sim 10^{6-8} \mathrm{~cm}^{-3}\right)$ clumps. The asymmetric CND distribution beyond its inner rim and the presence of also a component of high-velocity $\left(v \sim \pm 300 \mathrm{~km} \mathrm{~s}^{-1}\right)$ ionised gas, suggest either a transient origin of the CND (produced by e.g. a gravitationally captured, tidally stretched cloud; Quinn et al. 1985; Zhao et al. 1995), or an energetic disruption of a stable disc (e.g. produced by the impact of the Sgr A East supernova remnant upon the disc; Maeda et al. 2002; Rockefeller et al. 2005). The mass inflow rate of CND gas migrating into the central parsec is $\sim 10^{-2}-10^{-4}$ $\mathrm{M}_{\odot} \mathrm{yr}^{-1}$ (Gusten et al. 1987; Jackson et al. 1993; Vollmer et al. 2002; Genzel et al. 2010), while the accretion rate onto the accretion shock (far less gas may actually make it to Sgr A* event horizon) is estimated to be few $\sim 10^{-6} \mathrm{M}_{\odot} \mathrm{yr}^{-1}$ (Cuadra et al. 2008; Baganoff et al. 2003; Xu et al. 2006). This strongly suggests that the present dim period in Sgr A*'s life might be just a temporary phenomenon. The CND provides a reservoir of gas that can be accreted if local processes that cause angular momentum loss can bring material inwards. The ionised "arms" of Sgr A West (the so called mini-spiral) are possibly infalling fragments of the CND that can, on a time scale of $\sim 10^{2-3}$ years, give rise to energetic accretion events.

\subsection{The central cluster of young stars}

Within the central parsec, there are several populations of stars. The nuclear cluster, forming $98 \%$ of the observed stars and composed of old $\left(>10^{9} \mathrm{yr}\right)$, late-type giants and helium burning stars, plus $\sim 200$ young $\left(t=6 \pm 2 \times 10^{6} \mathrm{yr}\right.$ ) stars (one of the richest massive star forming regions in the entire Galaxy), forming a strongly warped disc (Genzel et al. 2003; Paumard et al. 2006; Tanner et al. 2006; Lu et al. 2009; Gillessen et al. 2009; see Genzel et al. 2010 for an overview). The tidal shear of the Galactic black hole would impede normal star formation, so for a long time, the presence of the young stars around it gave rise to the "paradox of youth" (Ghez et al. 2003).

It is now believed that they could have formed in situ (see Genzel et al. 2010 for a review) in a disc either rapidly produced by a massive cloud plunging into Sgr A* (although it seems strange that a molecular cloud reaches the GC at small angular momentum) or slowly building up, but with star formation quenched, until a trigger is reached. Once the disc is formed, rapid dissipation and cooling of fragmented parts of the disc might occur. These clumps would then efficiently form stars (Nayakshin et al. 2007; Hobbs \& Nayakshin 2009; Gualandris et al. 2012). Monte 
Carlo simulations show that, in this case, the amount of gas accreted through the inner radius of the disc is large enough to provide $\mathrm{Sgr} \mathrm{A}^{*}$ with the gas to radiate near its Eddington limit throughout the star-formation episode. The ages of the young stars suggest that this event occurred $\sim 6 \pm 2 \times 10^{6} \mathrm{yr}$ ago, a time-scale compatible with the formation of the Fermi bubbles and the launch of the EMR. Simulations also show that a cloud captured by Sgr A* is expected to form a sheared and asymmetric circumnuclear disc very closely resembling the observed CND (although the likelihood of such an event needs to be demonstrated). Whichever the process that created it, the presence of a massive disc and star formation in the immediate vicinity of the black hole would necessarily be accompanied by a high accretion rate onto Sgr A*. Therefore this star formation would be accompanied by strongly enhanced AGN activity (which, in fact, could have been the trigger for the star formation). Thus, the detailed study of Sgr A*'s environment reveals further hints of a possible glorious past for Sgr A*.

\subsection{Fermi bubbles and evidence for Sgr A*'s jets}

A pair of large scale structures, extending up to 50 degrees (i.e. $\sim 10 \mathrm{kpc}$ ) above and below the Galactic plane and with a width of about $40^{\circ}$, have been recently detected, at $\gamma$-ray energies, above a few $\mathrm{GeV}$, thanks to Fermi data (Su et al. 2010). These so-called "Fermi bubbles" are centered on the core of the Milky Way, they have approximately uniform gamma-ray surface brightness with sharp edges. The bipolar morphology and sharp edges suggest that the Fermi bubbles originated by some large episode of energy injection in the GC (e.g., jets originating from AGN activity or nuclear starburst). The bubbles have a luminosity of $4 \times 10^{37} \mathrm{erg} \mathrm{s}^{-1}$, they extend to $\sim 10 \mathrm{kpc}$ outside the Galactic disc and have an estimated energy content of the order of $\sim 10^{55}$ erg (Bland-Hawthorn \& Coen 2003; Su et al. 2010). The Fermi bubbles have a hard spectrum extending up to $100 \mathrm{GeV}$ and they seem to be associated with large scale structures observed in X-rays with ROSAT (BlandHawthorn \& Cohen 2003) and with an excess of radio emission found in WMAP data (the so called Galactic haze; Dobler \& Finkbeiner 2008). New Planck data have recently confirmed the microwave haze to have a morphology consistent with the Fermi bubbles. The Planck data reveal a hard microwave spectrum consistent with hard synchrotron radiation and excluding free-free emission (Planck collaboration 2012).

The mechanism producing the bubbles is still quite uncertain. Su et al. (2010) discussed a possible starburst phase in the GC a few million years ago. A modest, but constant, injection of $\sim 10^{39} \mathrm{erg} \mathrm{s}^{-1}$ of cosmic rays to the halo is today inferred by the IR luminosity and $\gamma$-ray emission from the CMZ (Crocker et al. 2011). Integrating this emission over $\sim 10^{9} \mathrm{yr}$ would produce the correct energy budget (Crocker \& Aharonian 2011), however a mechanism to confine the particles for such a long time would be required. Alternatively, the Fermi bubbles, X-ray structure and microwave haze might all be produced by an intense AGN phase, with 
Sgr $A^{*}$ accreting close to the Eddington limit, a few $10^{6} \mathrm{yr}$ ago (Zubovas et al. 2011). Recently Su \& Finkbeiner (2012) reported a gamma ray cocoon feature, in the southern Fermi bubble, and they produced evidence for a jet-like feature along the cocoon axis with a luminosity of $L_{1-100 \mathrm{Gev}} \sim 5.5 \pm 0.45$ and $1.8 \pm 0.35 \times 10^{35}$ $\mathrm{erg} \mathrm{s}^{-1}$ for the cocoon and jet, respectively. If this finding is confirmed, this would strongly support an accretion phase onto Sgr A* that would be therefore associated with a relativistic jet and outflows/winds as generally observed in AGN and stellar mass black holes (Antonucci 1993; Fender et al. 2004; Ponti et al. 2012). For deeper insight into the physics associated with the Fermi bubbles we point the reader to the contribution, in this same volume, by M. Su and R. Crocker.

On a much smaller scale of a few parsec, a faint, collimated linear structure observed at radio wavelength has been recently claimed by Yusef-Zadeh et al. (2012). This feature seems to arise from Sgr $\mathrm{A}^{*}$ and to interact with the ionised and molecular material orbiting the supermassive BH. Yusef-Zadeh et al. (2012) associate this linear structure to a jet possibly originating a few hundred years ago. Deeper observations are necessary to confirm this feature. A different linear feature pointing to the location of Sgr A* has been noted in X-ray images of the central parsec (Muno et al. 2008; Li et al. 2012). The X-ray emission is interpreted as being synchrotron emission produced by a population of highly relativistic electrons created by the shock at the collision point between the jet and the Eastern Arm of the mini-spiral inside the CND (Li et al. 2012).

\section{Is the GC Fe K $\alpha$ emission the echo of a past Sgr A* flare?}

The discovery of the $\mathrm{Fe} \mathrm{K} \alpha$ line and hard X-ray emission from several massive MC in the CMZ placed on solid ground the idea of this emission is being produced by reflection of a past GC flare (Sunyaev et al. 1993; Koyama et al. 1996). Deep ASCA observations of Sgr B2, the most massive MC complex in the CMZ, showed many spectral features reminiscent of an X-ray reflection nebula (see Fig. 4) and 1), such as: i) strong and extended $\mathrm{Fe} \mathrm{K} \alpha$ emission with $\sim 1-2 \mathrm{keV} \mathrm{EW}$; ii) a low-energy cut-off below $\sim 4 \mathrm{keV}$; iii) a sharp flux drop consistent with the presence of an Fe K edge (Koyama et al. 1996; Murakami et al. 2000). Not only spectral, but also morphological evidences has been accumulating. For example, in the Sgr B complex, Murakami et al. (2000) observed the peak of the extended $6.4 \mathrm{keV}$ emission from Sgr B2 being shifted toward the GC by about $\sim 1-2$ arcmin compared to the molecular mass distribution, thus suggesting the presence of an irradiating X-ray source located in the direction of the GC. Assuming that $\mathrm{Sgr} A *$ is the illuminating source, they estimated that, a few hundred years ago, it had to be orders of magnitudes brighter than now, with a 2-10 keV luminosity, $L_{2-10} \sim 3 \times 10^{39}(d / 100 p c)^{2}$ $\mathrm{erg} \mathrm{s}^{-1}$. A subsequent long Chandra observation confirmed these findings (allowing also the detection of the weaker Fe $\mathrm{K} \beta$ emission line) and finally, owing to the excellent imaging resolution, the Chandra data ruled out the possibility that the 6.4 

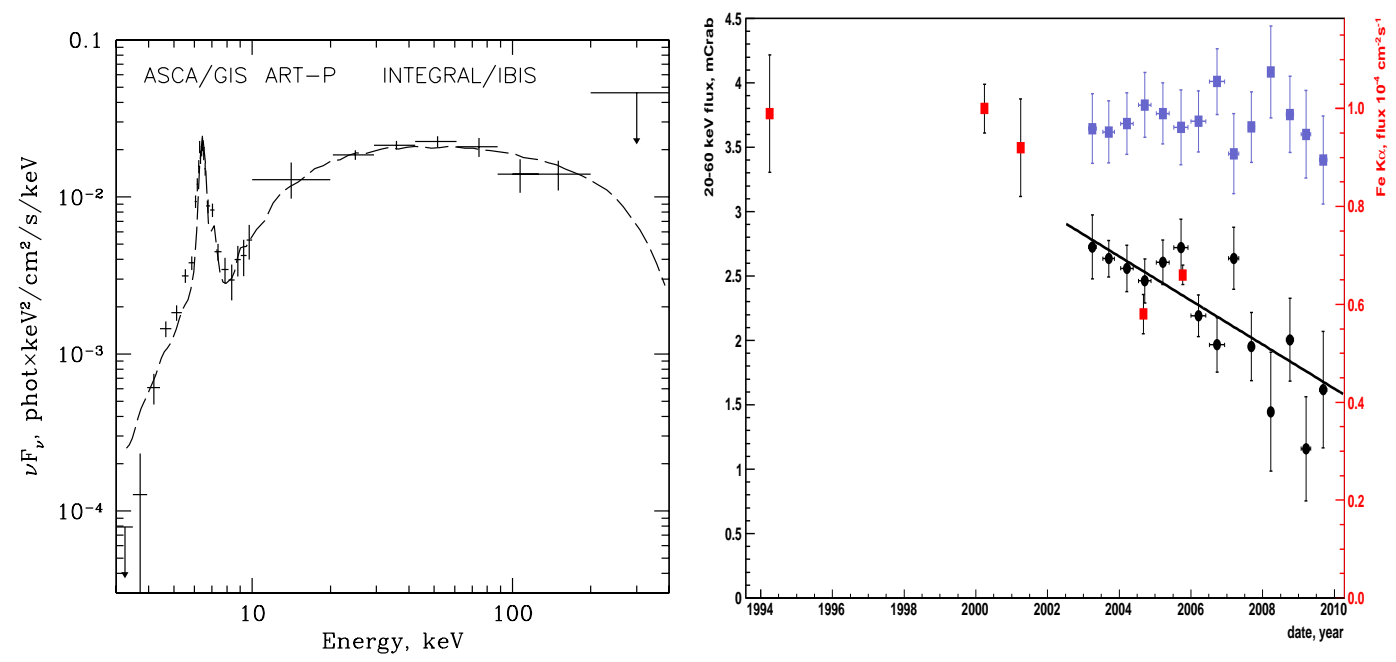

Fig. 4 (Left panel) ASCA, ART - P and INTEGRAL broad band X-ray spectrum of Sgr B2 (1 $\sigma$ error bars and $2 \sigma$ upper limits). The dashed line shows the best fit reflection model convolved with the instrumental resolution. [Figure courtesy of Revnivtsev et al. (2004)] (Right panel) INTEGRAL light curve of the corrected 20-60 keV Sgr B2 flux in mCrab (black circles) and of the secondary calibrator, Ophiuchus cluster (light blue squares). Superimposed (red squares) are the Fe K $\alpha$ line fluxes from Inui et al. (2009). A chi-squared test favors a linearly decreasing flux over a constant at the $\sim 4.8 \sigma$ level. [Figure courtesy of Terrier et al. (2010)]

$\mathrm{keV}$ emission was due to unresolved point sources, which contribute less than $3 \%$ of the diffuse Fe $\mathrm{K} \alpha$ emission (Murakami et al. 2001).

The combination of INTEGRAL, ASCA and GRANAT data on Sgr B2, allowed the detection, for the first time, of the Compton hump and the characterisation of the broad band 2-200 keV spectrum (see Fig. (4), which is well fitted by scattered and reprocessed radiation in a cloud of cold gas with abundances about twice Solar (Revnivtsev et al. 2004). Moreover, Revnivtsev et al. (2004) found no significant variability of the $\mathrm{Fe} \mathrm{K} \alpha$ during the 1993-2001 period. They excluded an internal source hypothesis and, based on the intensity of the broad-band spectrum and the cloud column density, they estimated a high luminosity, $L_{2-10 / 2-200} \sim 0.5 / 1.5 \times$ $10^{39}(r / 10 p c)^{-2}(d / 100 p c)^{2} \mathrm{erg} \mathrm{s}^{-1}$ of the primary illuminating source, active for many years. Such luminosity and period of activity are higher (even higher than the Eddington luminosity for $\mathrm{M}_{\mathrm{BH}}=10 \mathrm{M}_{\odot}$ ) and much longer than the typical values observed in X-ray binaries, thus disfavouring these as possible candidates.

$\mathrm{Fe} \mathrm{K} \alpha$ emission has also been detected in a number of other MC, such as Sgr C (Murakami et al. 2001; Sidoli et al. 2001) and small regions in the Sgr A complexes, such as the G0.11-0.11 cloud located close to the radio arc and the bridge (Bamba et al. 2002; Park et al. 2004). All these Fe K $\alpha$ clumps are associated with molecular complexes and they have $\mathrm{EW}_{\mathrm{FeK} \alpha} \sim 1-2 \mathrm{keV}$, in agreement with a re- 
flection origin. The increased Suzaku sensitivity allowed Koyama et al. (2007a) to detect also the Ni $\mathrm{K} \alpha$ emission from several molecular complexes and to discover, in the Sgr B complex, two other X-ray nebulae, M0.74-0.09 and M0.51-0.10 (the latter coincident with Sgr B1). These molecular complexes show a decay of the $\mathrm{Fe}$ $\mathrm{K} \alpha$ emission similar to the one observed in Sgr B2, moreover if illuminated by Sgr A* they would require similar luminosity, suggesting that they might be illuminated by the same source irradiating Sgr B2 (Koyama et al. 2007b; Nobukawa et al. 2008; but see also Yusef-Zadeh et al. 2007).

\subsection{Alternative mechanisms to produce FeK $\alpha$ emission from MC}

$\mathrm{X}$-ray irradiation can induce $\mathrm{Fe} \mathrm{K} \alpha$ line emission by removal of a $\mathrm{K}$-shell electron rapidly followed by an electronic transition from the L shell to fill the vacancy (see (2). However, the same process can also be produced by collisional ionisation induced by accelerated particles, such as low-energy cosmic ray electrons as well as protons/ions. Moreover, the energetic particles, diffusing in dense neutral matter, do produce nonthermal X-rays by atomic collisions as they slow down by ionisation and radiative energy losses (non-thermal bremsstrahlung), thus producing a hard X-ray continuum emission (Valinia et al. 2000; Tatischeff 2003; 2012).

The physical conditions in the GC are exceptional (see $\$$ ) and make it a special place as regard to particle acceleration. Bright non-thermal radio filaments trace magnetic fields up to a $\mathrm{mG}$ (Ferriere 2009) and large-scale non-thermal emission suggest the average field to be at least $50 \mu \mathrm{G}$ (Crocker et al. 2010). Three of the most massive young star clusters in the Galaxy lie in the CMZ, and intense star formation is taking place there at several sites. It is possible that the density of lowenergy cosmic rays is larger in the central regions than in the rest of the Galaxy (Berezinskii et al. 1990; Dogiel et al. 2002).

Cosmic ray electrons: The presence of strong non-thermal radio filaments in the vicinity of several MC (Yusef-Zadeh et al. 2002; 2007) makes it reasonable to consider the presence of a large density of cosmic ray electrons there. This is also supported by the observation of diffuse low-frequency radio emission in the GC region (LaRosa et al. 2005) and the high estimates of the ionisation rate compared to the values obtained in the Galactic disc (Oka et al. 2005). It has also been suggested (Yusef-Zadeh et al. 2007) that the heating of MC induced by the interaction with cosmic ray electrons might explain the long-standing problem of the origin of the high temperature of the MC within the CMZ (Morris \& Serabyn 1996). Several authors (Valinia et al. 2000; Tatischeff 2003; 2012) studied in detail the production of non-thermal lines and X-ray continuum by the interaction of cosmic ray electrons with neutral ambient gas. In most cases the EW of the $\mathrm{Fe} \mathrm{K} \alpha$ line is found to be lower than $\sim 0.3-0.5 \times\left(A_{\mathrm{Fe}} / A_{\odot}\right) \mathrm{keV}$ (where $A_{\mathrm{Fe}} / A_{\odot}$ is the Fe fractional abundance compared to Solar) and never higher than $\sim 0.6 \times\left(A_{\mathrm{Fe}} / A_{\odot}\right) \mathrm{keV}$, and the continuum radiation should be generally hard $(\Gamma<1.4)$. The production of $\mathrm{Fe}$ $\mathrm{K} \alpha$ photons is relatively inefficient. The $\mathrm{Fe} \mathrm{K} \alpha$ total luminosity from the $\mathrm{CMZ}$ is 
$\gtrsim 6 \times 10^{34} \mathrm{erg} \mathrm{s}^{-1}$ (Yusef-Zadeh et al. 2007), thus requiring a power $\gtrsim 2 \times 10^{40} \mathrm{erg}$ $\mathrm{s}^{-1}$ in cosmic ray electrons (assuming Solar metallicities), which is comparable to that contained in cosmic ray protons in the entire Milky Way (Tatischeff et al. 2012).

Cosmic ray ions/protons: Alternatively, subrelativistic ions propagating through MC can radiate (through inverse bremsstrahlung from the fast ions and classical bremsstrahlung from the secondary knock-on electrons) in the hard X-ray domain and create $\mathrm{Fe} \mathrm{K} \alpha$ vacancies (Dogiel et al. 2009; 2011; Tatischeff et al. 2003; 2012). Dogiel et al. (2009) proposed that the tidal disruption and subsequent accretion of stars by the central BH (suggested to happen at a rate of one every $\sim 10^{4} \mathrm{yr}$; Syer \& Ulmer 1999) could produce the required rate (basically constant on time-scales of centuries) of subrelativistic protons to induce the observed $\mathrm{Fe} \mathrm{K} \alpha$ emission. For strong shock acceleration of non-relativistic particles and strong particle diffusion in the cloud, the expected Fe $\mathrm{K} \alpha \mathrm{EW}$ is expected to be $\sim 0.6-0.8 \times\left(A_{\mathrm{Fe}} / A_{\odot}\right) \mathrm{keV}$, with an associated continuum having spectral photon index $\Gamma \sim 1.3-2$. However, fast ions with a soft source spectrum can produce very large EW ( $\gg 1 \mathrm{keV})$ and steep $(\Gamma \gg 2)$ power-law continuum (Dogiel et al. 2011; Tatischeff et al. 2012). In this scenario the $\mathrm{Fe} \mathrm{K} \alpha$ line width is expected to be several tens of $\mathrm{eV}$, about one order of magnitude wider than for X-ray reflection or cosmic ray electron scenario (Tatischeff et al. 1998; Dogiel et al. 2009). In fact, the X-ray lines produced by collisions of ions heavier than $4 \mathrm{He}$ can be shifted by several tens of eV, significantly broadened and split up into several components (Garcia et al. 1973; Tatischeff et al. 1998). For example, the $\mathrm{Fe} \mathrm{K} \alpha$ line produced by impacts of $\mathrm{O}$ ions of $1.9 \mathrm{MeV} /$ nucleon is blueshifted by $\sim 50 \mathrm{eV}$ in comparison with the one produced by $5-\mathrm{MeV}$ proton impacts, and has a FWHM of $\sim 100 \mathrm{eV}$ (see Garcia et al. 1973, Fig. 3.55)

As another alternative, Bykov (2003) proposed that subrelativistic particles are produced by fast-moving knots resulting from supernova explosions. In fact, fast moving supernova ejecta interacting with dense MC provide a fast conversion of kinetic energy into IR and X-ray radiation with a hard $(\Gamma \sim 0-1.5)$ spectrum and Fe $\mathrm{K}$ lines $(6.4$ and $6.7 \mathrm{keV})$ with total $\mathrm{EW} \sim 0.5-0.6 \mathrm{keV}$. Just $\sim 3$ supernova remnants of age less than $\sim 10^{3} \mathrm{yr}$ in the GC region could provide the required number of fast moving knots (Bykov 2003).

\subsection{A Christmas tree around Sgr A*}

A major step forward has been the detection of variability from $\mathrm{Fe} \mathrm{K} \alpha$ emitting clouds. The first such detection was reported by Muno et al. (2007), who showed $\mathrm{a} \sim 2-4 \sigma$ continuum variability from filamentary $(\sim 0.3 \times 2 \mathrm{pc})$ regions that are dominated by $\mathrm{Fe} \mathrm{K} \alpha$ emission and coincident with molecular structures near Sgr A (Muno et al. 2007). Fast variability is a key factor to discriminate X-ray reflection nebulae from $\mathrm{Fe} \mathrm{K} \alpha$ emission induced by cosmic ray interactions with MC (Valinia et al. 2000; Yusef-Zadeh et al. 2002; 2007; Bykov 2002; Dogiel et al. 2009). Fast $\mathrm{Fe} \mathrm{K} \alpha$ or hard $\mathrm{X}$-ray continuum variability, in fact, rules out the 
cosmic ray proton scenarios and is barely consistent with the cosmic ray electron models. The variations observed by Muno et al. (2007) in the Sgr A complex could be produced by an X-ray binary of $L_{X} \sim 10^{37} \mathrm{erg} \mathrm{s}^{-1}$ at a distance of $\sim 7 \mathrm{pc}$. If, instead, the irradiating source is Sgr A* (thus at, or further away than, the projected distance of $14 \mathrm{pc}$ ) then $L_{X} \gtrsim 10^{38} \mathrm{erg} \mathrm{s}^{-1}$. Muno et al. (2007) suggested that the flare illuminating these MC in Sgr A might be different from the one irradiating Sgr B2. If these MC were located at their projected distances, such an outburst, in fact, would have occurred $t \gtrsim 60 \mathrm{yr}$ or $t \sim 20 \mathrm{yr}$ ago, before the advent of wide field X-ray monitors, thus it could have been missed (Muno et al. 2007).

Collecting data from ASCA, Suzaku, Chandra and XMM-Newton, Inui et al. (2009) and Koyama et al. (2008) observed a decline, of the order of $\sim 60 \%$ in $\sim 10 \mathrm{yr}$, of the $\mathrm{Fe} \mathrm{K} \alpha$ line flux from both the Sgr B2 and Sgr B1 regions. Using INTEGRAL data, Terrier et al. (2010) measured a $4.8 \sigma$ variation of the $20-60 \mathrm{keV}$ (the energy of the Compton hump) flux from Sgr B2, starting to decline after the year 2000 (black points in Fig. 4). This confirmed, without inter-calibration effects, the $\mathrm{Fe} \mathrm{K} \alpha$ decay (red points in Fig. (4) previously observed. The observed decay time $\left(t_{d}=8 \pm 1.7 \mathrm{yr}\right)$ is compatible with the light crossing time of the MC core, consistent with reflection but inconsistent with an electron cosmic ray scenario. The spectral index of the illuminating power-law source is found to be $\Gamma=2 \pm 0.2$. Parallax measurements of the distance of Sgr B2 place it (assuming it is on a nearly circular Galactic orbit) $130 \pm 60$ pc closer to us than Sgr A* (Reid et al. 2009; Ryu et al. 2009; but see also Molinari et al. 2011). This suggests a mean luminosity for the Sgr A* outburst of $L_{X}=1.5-5 \times 10^{39} \mathrm{erg} \mathrm{s}^{-1}$, occurring $t=100_{-25}^{+55} \mathrm{yr}$ ago (Terrier et al. 2010). New Suzaku observations obtained in fall 2009, when compared to previous observations obtained in fall 2005, confirm Sgr B's fading (Terrier et al. 2010) detecting, in $4 \mathrm{yr}$, a reduction in $\mathrm{Fe} \mathrm{K} \alpha$ flux and hard (8-10 keV) continuum by a factor 1.9-2.5 from both Sgr B2 and M0.74-0.09 (Nobukawa et al. 2011). In particular the variations of these two molecular cores decrease in a synchronised way. The correlation of the variability between the two MC indicates that they share a common external reflection origin (Nobukawa et al. 2011).

The Sgr A and Sgr B complexes are not the only ones to display Fe K $\alpha$ variations. The comparison between a Suzaku observation in January 2006 and previous ASCA and Chandra observations suggested variability of the different $\mathrm{Fe} \mathrm{K} \alpha$ clumps in the Sgr C complex, all having $\mathrm{EW}_{\mathrm{FeK} \alpha} \sim 2.0-2.2 \mathrm{keV}$ and clear Fe $\mathrm{K} \beta$ (Nakajima et al. 2009). In particular, the clump M359.38-0.00 was not previously observed to emit Fe K $\alpha$ photons. Moreover, the Fe K $\alpha$ emission from M359.43-0.07 appeared shifted compared to previous ASCA observations, suggesting a reflection origin of this emission (Murakami et al. 2001). Interestingly, the M359.38-0.00 and M359.470.15 clumps correspond to molecular complexes with different line of sight velocities, which are thought to belong to two different arms of the CMZ MC population (see Fig. 3, Sofue 1995; Sawada et al. 2004; Nakajima et al. 2009). This indicates that they most probably have very different line-of-sight distances compared to Sgr A*. This seems to suggest that these two clumps are irradiated by two different beams of radiation, for example by two different flares or by two phases of the same long flare. 


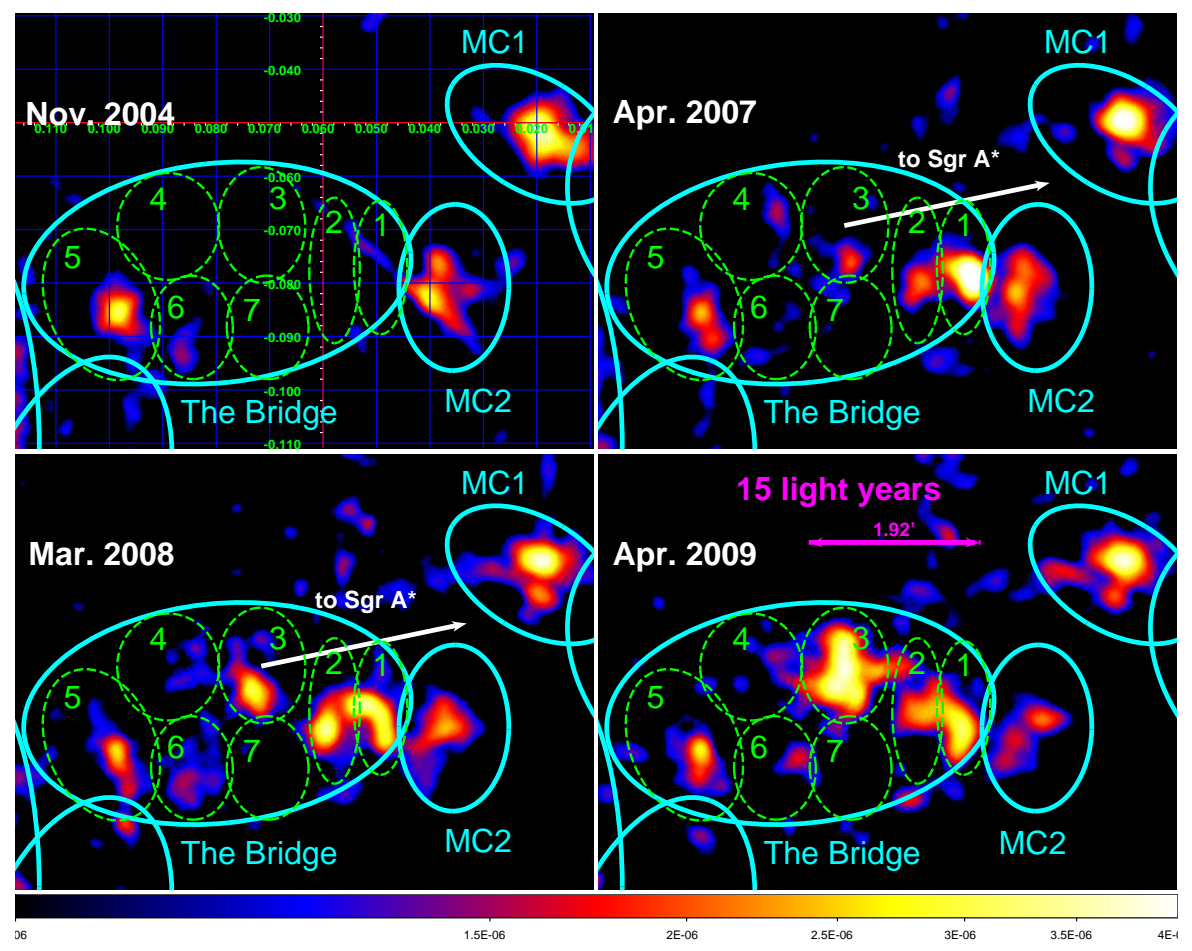

Fig. $5 \mathrm{Fe} \mathrm{K} \alpha$ continuum-subtracted mosaic image of the different EPIC-pn observations of the bridge region. A brightening of regions 1, 2, 3 and 4 is clear. Such variation occurs in a time-scale of about 2-4 years, but on a spatial scale of about 15 light years. This apparent superluminal motion can be explained if the bridge MC is illuminated by a bright $\left(L>1.3 \times 10^{38} \mathrm{erg} \mathrm{s}^{-1}\right)$ and distant $(>15 \mathrm{pc}) \mathrm{X}$-ray source active for several years. The observation of the superluminal echo cannot be explained by either a single internal source or by low energy cosmic ray irradiation. It is also highly unlikely that the variation is produced by several uncorrelated sources. We note that the illumination starts in the Galactic west and propagates to the east, suggesting that the source is located in the direction of Sgr A*.

The long (more than $1 \mathrm{Ms}$ exposure) XMM-Newton monitoring campaign of the Sgr A complex (lasting for almost a decade), led to another important step forward. It allowed the first detection of highly significant $(\sim 4-13 \sigma) \mathrm{Fe} \mathrm{K} \alpha$ variations from several MC in Sgr A (Ponti et al. 2010) as well as the detection of a superluminal echo moving with apparent velocity of $v_{\text {app }} \sim 3 \mathrm{c}$ (see Fig. 5) from a molecular complex called the bridge (Gusten Downes 1980). The superluminal propagation of the $\mathrm{Fe} \mathrm{K} \alpha$ emission strongly suggests an external illuminating source and rules out models based on internal sources and/or cosmic rays (which would have to deal with superluminal motions!).

Contrary to the case of the Sgr B complex, where every clump is consistent with being produced by the same flare, the $\mathrm{Fe} \mathrm{K} \alpha$ emitting regions in Sgr A have very different light curves (Ponti et al. 2010; Capelli et al. 2012). In some MC the Fe 
$\mathrm{K} \alpha$ emission is rising, in others it is fading and in some it is constant (Ponti et al. 2010; Capelli et al. 2012). It is interesting to note that, as in Sgr B2, the Fe $\mathrm{K} \alpha$ emission from G0.11-0.11 (a MC located close to the radio arc) is fading on a short time scale (comparable to the light crossing time of the MC). If located at its minimal distance from $\mathrm{Sgr} \mathrm{A}^{*}(25 \mathrm{pc})$ it would require $L_{X} \gtrsim 10^{39} \mathrm{erg} \mathrm{s}^{-1}$, $t \gtrsim 75 \mathrm{yr}$ ago (Ponti et al. 2010). These estimates would necessitate a flare of Sgr A* with a luminosity and a difference in time of the same order of magnitude as the one implied by Sgr B2 and B1. Moreover all three of these MC are fading at a rate comparable to the $\mathrm{MC}$ light crossing time. This seems to suggest that $\mathrm{Sgr} \mathrm{B} 1, \mathrm{~B} 2$ and G0.11-0.11 are illuminated by the same flare. On the contrary the bridge (see Fig. 5) shows a clear $(13 \sigma)$ increase of Fe $\mathrm{K} \alpha$ emission, while other $\mathrm{MC}$ are consistent with being constant over the XMM-Newton monitoring (Ponti et al. 2010). While the flare seems to be passing out of some clouds, it is entering others, or is still in the act of passing through others. The rise, fall, and dwell time scales will all be important for reconstructing the flare profile, and for deciding whether there have been multiple flares within the past several hundred years.

\subsection{Which source of irradiation?}

The very different variability behaviour might indicate that each one of these MC is responding to a different flare of, possibly, separate sources. The observation of a superluminal echo (Ponti et al. 2010) and the synchronised fading of the causally disconnected regions in the Sgr B complex (Nobukawa et al. 2011) constrain the mechanism of $\mathrm{Fe} \mathrm{K} \alpha$ production to be irradiation from a powerful external source.

As described in 1.1 only 3 sources in the central 1.2 square degrees have reached luminosities higher than $\mathrm{L}_{X} \gtrsim 10^{37} \mathrm{erg} \mathrm{s}^{-1}$ during the past few decades (Degenaard et al. 2012; Wijnands et al. 2006). The highest peak luminosities have been reached by 1A 1742-289 and GRO J1744-28 with $\mathrm{L}_{X} \sim 7 \times 10^{38} \mathrm{erg} \mathrm{s}^{-1}$ and

$\mathrm{L}_{X} \sim 3 \times 10^{38} \mathrm{erg} \mathrm{s}^{-1}$, respectively (Degenaard et al. 2012). Both these sources are $\mathrm{X}$-ray transients, thus active only for few weeks-months. No known X-ray binary undergoes such a long outburst with such high mean luminosity (Coriat et al. 2012) as the source irradiating the Sgr B complex. However, this does not exclude that bright X-ray transients, located close enough to a MC, might produce detectable $\mathrm{Fe}$ $\mathrm{K} \alpha$ echos. Moreover, it does not exclude a past outburst of an even more extreme version (with even longer recurrence time and higher luminosity) of the peculiar BH X-ray binary GRS1915+105 (a source accreting close to the Eddington rate for more than a decade; Fender \& Belloni 2004). Although this is possible, we believe that a flare from $\mathrm{Sgr} \mathrm{A}^{*}$ is more plausible and more parsimonious for explaining all of the variability throughout the GC region.

Alternative possibilities involve bright X-ray sources produced by either the passage of the shock wave, generated by the supernova explosion that gave birth to the Sgr A East remnant, over the supermassive BH (Maeda et al. 2002; Rockefeller et al. 2005) or the interaction between Sgr A East and the $50 \mathrm{~km} \mathrm{~s}^{-1}$ MC (Fryer et 
al. 2006). Fryer et al. (2006) performed three-dimensional hydrodynamic simulations of the evolution of Sgr A East and its interaction with surrounding medium (e.g. Sgr A* and the $50 \mathrm{~km} \mathrm{~s}^{-1} \mathrm{MC}$ ). They found that the passage of the remnant across Sgr $\mathrm{A}^{*}$ would have enhanced the accretion rate onto the $\mathrm{BH}$ by less than a factor of 2, thus not enough to explain the $\mathrm{Fe} \mathrm{K} \alpha$ emission from the CMZ. On the other hand, the first impact of the shock wave with the material in the $50 \mathrm{~km} \mathrm{~s}^{-1}$ MC would have produced a luminosity of $L_{2-200 \mathrm{keV}} \sim 10^{39} \mathrm{erg} \mathrm{s}^{-1}$, decreasing to $\sim 10^{36} \mathrm{erg} \mathrm{s}^{-1}$ in the following few centuries. The resulting light curve would have, therefore be predicted to have a sharp rise, followed by a very long decay time (a few centuries), dictated by the time-scale for dissipation of kinetic energy (Fryer et al. 2006). In this scenario the observed rapid and small amplitude $\mathrm{Fe} \mathrm{K} \alpha$ variability might be produced by the interaction of the Sgr A East shock with dense matter clumps in the $50 \mathrm{~km} \mathrm{~s}^{-1} \mathrm{MC}$. However, it seems difficult to reconcile the expected slow luminosity decay with the fast decrease of a factor of $\sim 3-4$ in 5-7 yr (Terrier et al. 2010; Nobukawa et al. 2011) observed in Sgr B2.

Before attempting to reconstruct the past activity in the GC, we summarise the main limitations and uncertainties associated with the tools that are used.

\subsubsection{Uncertainties on the luminosity estimates}

We try to estimate here the dominant source of uncertainty in the estimation of the primary source's past luminosity. Equation 1) (which assumes isotropic source emission and a cloud with $\left.\sigma_{T} \ll 1\right)$ provides a direct link between the observed $\mathrm{Fe} \mathrm{K} \alpha$ line flux (or upper limit) and luminosity of the source (e.g. Sgr A*), and is the main tool. The luminosity of the source depends linearly on the $\mathrm{Fe} \mathrm{K} \alpha$ line flux and inversely on the distance to the GC. Both are generally well known. The former to better than $10-15 \%$ and the latter to less than $5 \%$. A higher uncertainty is associated with the knowledge of the Fe abundance, $\delta_{F e}$, which however can be constrained from the $\mathrm{Fe} \mathrm{K} \alpha$ line $\mathrm{EW}$ and is expected to have fluctuations of a factor of less than a few. Larger uncertainties are associated with the determination of the cloud optical depth, $\tau_{T}$, and mainly the solid angle $\Omega$ of the cloud from the location of the primary source.

Solid angle: This latter parameter $\left(\Omega=A_{\text {cloud }} / 4 \pi d^{2}\right)$ depends on the ratio between the area exposed to the illuminating source $\left(A_{\text {cloud }}\right)$ and the square of the distance $(d)$ between the source and the cloud. Fig. 2 shows a large concentration of molecular matter along any direction of the CMZ. In particular, several clouds typically overlap along the line of sight, thus confounding the boundaries between the clouds. Each cloud is expected to move with approximately the same velocity. Thus, thanks to the molecular cloud data cubes that show the MC emission in each velocity range, we can separate the emission from each cloud along the line of sight and accurately measure the correct MC area. MC typically have complex shapes (see Fig. 21) and the area irradiated by the source might be different from the area projected on the image, however we expect this difference to be smaller than a factor of a few. 
The dominant uncertainty is associated with the line-of-sight component of the distance between the cloud and $\operatorname{Sgr} A^{*}$, to be $d_{l}$. Being the CMZ compact, we expect that for each CMZ cloud, $d_{l}$ is constrained between the projected distance and $\sim \pm 300 \mathrm{pc}$. This generally implies a very large uncertainty that can be as high as 1-2 orders of magnitude. Thus, knowledge of the line of sight distance between the MC and Sgr A*'s plane is key for accurate luminosity estimates. As discussed in $\$ 3$ there are several indications of coherent patterns in the MC distributions, from which distances might be derived. However, although these large molecular structures incorporate a significant part of the gas, a further effort is required in order to accurately associate each molecular clump with a line of sight distance. Other methods to determine $d_{l}$ are based on extinction measurements. Sources distributed uniformly around the GC will appear more extinguished if the $\mathrm{MC}$ in question is placed more in the foreground, while MC placed at the far side of the CMZ will contribute little or no absorption.

Ryu et al. (2009), assuming that the $6.7 \mathrm{keV}$ and the diffuse soft X-ray plasma (see 1.1 ) emission are uniformly distributed around the GC, measured the amount of absorption toward each molecular clump in the Sgr B complex. Seven different spectral components (with an even larger number of free parameters) contribute to the X-ray spectrum, and thus an exceptionally high X-ray spectral quality is required in order to constrain the various components without incurring degeneracies between the different parameters. Moreover, intensity fluctuations of the diffuse soft $\mathrm{X}$-ray plasma (which is known to have a patchy distribution) is likely another source of uncertainty. The distribution of stars in the central $\sim 1$ degree is much more uniform. Thus stellar counts in near-IR images can provide reliable constraints on the line of sight distance toward molecular clumps (see, e.g., Gusten \& Downes 1980; Glass et al. 1987). Sawada et al. (2004) proposed a different method based on the quantitative comparison between the 2.6-mm CO emission lines and $18-\mathrm{cm} \mathrm{OH}$ absorption lines. The GC is an intense and presumably axisymmetric source of diffuse, non-thermal emission at $18-\mathrm{cm}$, so $\mathrm{OH}$ absorption arises preferentially from clouds located in the foreground of the GC. On the other hand, $\mathrm{CO}$ emission samples the gas both in front and back of the continuum source equally (Sawada et al. 2004), thus (assuming that the lines are optically thin and that the physical and chemical properties of GC clouds are uniform) the $\mathrm{OH} / \mathrm{CO}$ ratio for any given cloud provides information about its line-of-sight distance.

The use of the angular dependence of the $\mathrm{EW}_{\mathrm{Fe}}$ on the angle $\theta$ between the direction of the incident radiation and scattered continuum (see $\$$ 2) may eventually provide another means of estimating the line-of-sight distances to the $\mathrm{Fe} \mathrm{K} \alpha$ emitting MC (see Capelli et al. 2012). However, a maximum variation of the $\mathrm{EW}_{\mathrm{Fe}}$ of $\sim 50 \%$ is expected, thus very solid estimates of MC abundances (producing EW variations of the order of several; see $\$ 2$ ) and $N_{H}$ (producing EW variations of at least $\sim 30 \%$; Matt et al. 2003) are required.

Because of physical interactions between the different molecular filaments and streamers, it is easier to estimate the MC distribution in the immediate vicinity of Sgr A* (within the central $15 \mathrm{pc}$ ). Coil et al. (2000), in fact, suggested that the 20 and $50 \mathrm{~km} \mathrm{~s}^{-1} \mathrm{MC}$ are located at less than $15 \mathrm{pc}$ from Sgr A*, just outside the 
CND, with the former in front and the latter a few pc behind Sgr A* (see also Ferriere 2012). Perhaps the most promising method for determining the line-of-sight distances of some of the MC containing $\mathrm{H}_{2} \mathrm{O}$ maser sources is based on VLBI parallax measurements. Reid et al. (2009) measured a distance from the Earth to Sgr $\mathrm{B} 2$ of $R_{0}=7.9_{-0.7}^{+0.8} \mathrm{kpc}$ and, assuming a circular orbit for Sgr B2, they estimated Sgr $\mathrm{B} 2$ to be $\sim 130 \mathrm{pc}$ in front of Sgr A*. Although this measurement roughly agrees with the location estimated by Ryu et al. (2009), Molinari et al. (2011) suggested an elliptical orbit for Sgr B2 which would be then located behind Sgr A*. At the moment, it seems that the line of sight distance of the molecular clumps is the major source of uncertainty in measuring the GC's past activity (influencing both the derived luminosity and time evolution of the event).

Column density. The other major source of uncertainty is in the determination of the cloud column density. Three main ways to determine the MC column densities $\left(N_{H}\right)$ have been used: 1$)$ The $N_{H}$ can be derived directly from high quality $\mathrm{X}$-ray spectra of each molecular clump. In fact, as long as the column density is lower than the Compton thick limit $\left(N_{H} \lesssim \sigma_{T}^{-1}=1.5 \times 10^{24} \mathrm{~cm}^{-2}\right)$ the MC absorption will leave its signature through a clear low energy cut off in the 2-10 keV X-ray spectrum (Comastri 2004). However, these measurements trace only the total $N_{H}$ along the line of sight, thus including foreground absorption plus the additional contributions from any $\mathrm{MC}$ along the line of sight that are not related to the $\mathrm{Fe} \mathrm{K} \alpha$ emitting cloud. Moreover, such measurements are affected by the presence of many additional spectral components (e.g. $6.7 \mathrm{keV}$ emission, soft X-ray plasma, weak point sources) contributing to the observed MC X-ray emission. However, a strong improvement is expected soon thanks to hard X-ray focussing telescopes (such as NuSTAR and Astro- $H$ ) that will enhance, by more than an order of magnitude, the detector sensitivity in the 10 to $80 \mathrm{keV}$ energy band. In fact, in optically thin clouds $(\tau \ll 1)$, the shape and intensity of the high energy reflection spectrum depends strongly (see the right panel of Fig. 1) on the cloud column density (particularly the Compton hump, see $\$ 2$. Combining the information from the low energy reflection cut off, the line intensity and the Compton hump shape, NuSTAR and Astro-H X-ray spectra will soon provide a more reliable $N_{H}$ estimate. 2) Alternatively, the $N_{H}$ can be measured through the intensity of molecular emission lines. Tsuboi et al. (1999) provided a formulism (assuming a CS to $N_{H}$ abundance $X(C S)=10^{-8}$; Irvine et al. $1987)$ to derive the cloud $N_{H}$ from the CS line intensity $\left(\mathrm{I}_{C S}\right): N_{H}=\frac{7.5 \times 10^{11} \times T_{e x} \times I_{C S}}{X(C S)}$, where $T_{e x}$ is the excitation temperature. The great advantage of this method is that it allows us to measure the $N_{H}$ associated with each single molecular clump. In fact, any intervening material is expected to move at a different velocity, thus the extremely high spectral resolution with which it is possible to measure MC lines allows us to slice the CMZ in different velocity components, and to select MC of interest. However, large uncertainties are associated with $T_{e x}$ and the $X(C S)$ factor, unless detailed modelling is performed. Moreover, at high column densities $\left(N_{H}>\right.$ several $\left.\times 10^{23} \mathrm{~cm}^{-2}\right)$ most molecular lines become self-absorbed and hence are no longer good $N_{H}$ estimators. 3) Molinari et al. (2010) measured the cloud $N_{H}$ using IR Herschel observations. They first estimated the opacity and dust temperature from a pixel-to-pixel fit on the $70-350 \mu \mathrm{m}$ data, using a dust model (Com- 
piegne et al. 2011; Bernard et al. 2010). Then, assuming constant dust properties, they converted the opacity to a hydrogen column density $\left(N_{H}=N_{H I}+N_{H 2}\right)$ using $\tau_{250} / N_{H}=8.8 \times 10^{-26} \mathrm{~cm}^{2} / H$. The gas to dust ratio used is valid for the Galactic disc. Uncertainties of a factor of a few are associated with these measurements. In particular, different metallicities with respect to the disc values, would lower the estimated column densities by a factor of a few. Moreover, as with the $N_{H}$ estimated from X-ray absorption, the column densities derived with this method integrate the effects of dust along the line of sight.

\subsection{Sgr A*'s X-ray emission history}

The study of the Fe K $\alpha$ emission from the MC of the CMZ shows strong indications of external irradiation, most probably due to $\mathrm{Sgr} \mathrm{A}^{*}$. We also note that: i) spatially connected structures (such as Sgr B1 and B2 or the bridge) tend to vary in brightness with a coherent pattern; ii) molecular complexes that appear to be located close together, but display different $\mathrm{Fe} \mathrm{K} \alpha$ light curves (such as the bridge, MC1, MC2 and G0.11-0.11; Ponti et al. 2010), belong to molecular complexes moving at different line-of-sight velocities. The latter point indicates that these molecular complexes are actually well separated, in three dimensions, despite being close together on the plane of the sky. The different light curves observed from these complexes could then be tracing either different flares or different parts of the same long flare. Moreover, we note that smaller MC generally vary on faster time-scales (with the variation fronts usually propagating close to, or higher than, the speed of light) compared to larger MC. The latter varying on a time scale being typically of the order of the cloud light crossing time. This suggests that the $\mathrm{Fe} \mathrm{K} \alpha$ emission is induced by irradiation by an external source that is variable on both long and short time-scales. Such variability behaviour is usually observed in, for example, sources powered by accretion onto compact objects (Belloni \& Hasinger 1990; McHardy et al. 2006; Koerding et al. 2007; Ponti et al. 2012).

Constraints on the GC's past activity are given by the existence of MC that emit $\mathrm{Fe} \mathrm{K} \alpha$ and by those clouds that are quiet. Only upper limits to the $\mathrm{Fe} \mathrm{K} \alpha$ emission from the MC closest to $\mathrm{Sgr} \mathrm{A}^{*}$ (the $\mathrm{CND}$, the 20 and $50 \mathrm{~km} \mathrm{~s}^{-1} \mathrm{MC}$; Coil et al. 2000) are observed. If, indeed, the $50 \mathrm{~km} \mathrm{~s}^{-1} \mathrm{MC}$ is located 5-10 pc behind Sgr A* (Coil et al. 2000), then from the estimated column density and the upper limit on the line intensity, we can constrain the mean luminosity of Sgr A* to be lower than $L_{\mathrm{SgrA} *} \lesssim 8 \times 10^{35} \mathrm{erg} \mathrm{s}^{-1}$ in the past $60-90 \mathrm{yr}$ (Ponti et al. 2010). The observation of approximately constant $\mathrm{Fe} \mathrm{K} \alpha$ emission during the past decade, from molecular complexes with sizes smaller than a parsec, indicates that the flare illuminating those clouds lasted more than several years. Moreover, the observation of both a rising and

fading phase from some molecular complexes (Ponti et al. 2010; Capelli et al. 2011) suggests, not surprisingly, some degree of variability of the illuminating source. In fact, the light curves of accretion powered sources are well known to be variable 
(Belloni \& Hasinger 1990; McHardy et al. 2006; Koerding et al. 2007; Ponti et al. 2012).

The uncertainty in the line of sight distance toward the different molecular clouds in the CMZ currently prevents us from putting any firm constraint on the flare history, apart from knowing that Sgr $\mathrm{A}^{*}$ underwent one or more flares with luminosities of the order of $10^{39} \mathrm{erg} \mathrm{s}^{-1}$ or higher, between $\sim 100$ and $\sim 1000$ years ago (and possibly fainter ones). Assuming that Sgr B2 is located $\sim 130 \mathrm{pc}$ in front of Sgr $\mathrm{A}^{*}$, then the flare must have ended $\sim 100 \mathrm{yr}$ ago. The Fe K $\alpha$ evolution of G0.110.11 suggests that this cloud is illuminated by the same phase of the flare currently illuminating Sgr B2. Further observations will allow this hypothesis to be tested. Actually, if there is just a single (or a handful) illuminating source, we expect that eventually every MC will reflect the same light curve (convolved with the MC dimension). Therefore, it will eventually be possible to measure the delay of the light curve and hence determine the line of sight distance for each MC. Alternatively, if the line-of-sight to the clouds is known, it will be possible to triangulate the position of the primary source. The light echos provide us with the fantastic opportunity to scan the CMZ and determine its three dimensional distribution.

Although a precise number has not yet been measured, about one third of the $\mathrm{MC}$ in the CMZ are Fe K $\alpha$ active. This suggests that in the past $\sim 10^{3} \mathrm{yr}$ (the CMZ light crossing time), $\mathrm{Sgr}^{*}$ was active for a significant fraction of the time. Longer monitoring will clarify if the higher activity was made of several sharp excursions from quiescence to a short lived bright state, or if it involved a more gentle evolving and a longer period of activity. The constraints are still so weak that a single long period of activity is still consistent with the observations (Ponti et al. 2010, but see Capelli et al. 2012). New observing campaigns will provide stronger constraints, allowing us to learn much more about Sgr A*'s past activity.

Is it possible to constrain Sgr A*'s luminosity further in the past? To answer this question, Cramphorn \& Sunyaev (2002) studied the Fe K $\alpha$ emission from all the MC in the Galactic disc. None of the MC they observed showed any evidence of $\mathrm{Fe} \mathrm{K} \alpha$ emission. This allowed the authors to put several upper limits on Sgr A*'s historical luminosity. Sgr A*'s average luminosity was found to be lower than $\sim 10^{-(3-4)} \mathrm{L}_{\text {Edd }}$ since $\sim 4 \times 10^{4} \mathrm{yr}$ ago. Obviously the disc of the Milky Way is not completely filled with molecular gas, so gaps in the $\mathrm{CO}$ distributions leave unconstrained periods as long as $2-4 \times 10^{3}$ yr. To extend this study even further, Cramphorn \& Sunyaev (2002) investigated also the more extended HI distribution, allowing them to constrain Sgr A*'s past activity to $\mathrm{L}_{S g r A *}<10^{-2} \mathrm{~L}_{\mathrm{Edd}}$, in the last $\sim 1 \times 10^{5} \mathrm{yr}$.

To extrapolate even further into the past, different types of tracers of Sgr A*'s luminosity are necessary. The presence of the Fermi bubble, the EMR and the disc of young stars within the central parsec, suggest that $\mathrm{Sgr} \mathrm{A}^{*}$ was radiating close to the Eddington limit $\sim 5-6 \times 10^{6}$ years ago. 


\subsection{Is all the Fe K $\alpha$ emission due to Sgr A*'s flares?}

The measurements of fast Fe $\mathrm{K} \alpha$ flux variations (Inui et al. 2009; Terrier et al. 2010; Nobukawa et al. 2011; Ponti et al. 2010; Capelli et al. 2011; 2012) and even the super-luminal propagation of the X-ray echo (Ponti et al. 2010) have excluded a cosmic ray proton/ion origin for this variable component ( $\mathrm{Fe} \mathrm{K} \alpha$ emission induced by cosmic ray electrons might vary on years timescales; Yusef-Zadeh et al. 2012) and cosmic ray electron and internal source for the super-luminal one. This implies that at least part of the $\mathrm{Fe} \mathrm{K} \alpha$ radiation has to be produced by a bright external source, most probably Sgr A* (see 4.3. However, is it plausible to think that all Fe $\mathrm{K} \alpha$ radiation from $\mathrm{MC}$ of the $\mathrm{CMZ}$ is induced by $\mathrm{Sgr} \mathrm{A} *$ ?

Clear signatures for cosmic ray ion induced $\mathrm{Fe} \mathrm{K} \alpha$ emission have been discovered in molecular gas around the Arches cluster (Wang et al. 2006; Tsujimoto et al. 2007; Capelli et al. 2011; Tatischeff et al. 2012). The prominent (EW $1.2 \mathrm{keV}) \mathrm{Fe}$ $\mathrm{K} \alpha$ emission has, in fact, been observed to be constant over the past 8 yr (Capelli et al. 2011). Moreover the $\mathrm{Fe} \mathrm{K} \alpha$ emission is not well correlated with the molecular gas, forming, instead, a bow shock around the cluster (Wang et al. 2006). The Arches cluster is one of the richest and most densely packed massive $\left(M \sim 5 \times 10^{4}\right.$ $\mathrm{M}_{\odot}$; Harfst et al. 2010) star clusters in the Milky Way and is currently moving at highly supersonic speed $\left(v_{\text {Arch }} \sim 200 \mathrm{~km} \mathrm{~s}^{-1}\right)$ relative to the bulge stars. Figer et al. (2002) and Wang et al. (2006) found that the cluster bow shock is interacting with a MC and Tatischeff et al (2012) computed that the shock (assuming a reasonable particle acceleration efficiency of $\sim 3-10 \%$ ) could supply enough cosmic ray power to reproduce the $\mathrm{Fe} \mathrm{K} \alpha$ emission. Therefore not every $\mathrm{Fe} \mathrm{K} \alpha$ active cloud is illuminated by a flare from Sgr A*.

A deep Suzaku observation of the molecular cloud G0.162-0.217 revealed a weak $\mathrm{Fe} \mathrm{K} \alpha$ line with $\mathrm{EW}_{\mathrm{FeK} \alpha} \sim 0.2 \mathrm{keV}$ (Fukuoka et al. 2009). The cloud is located at the south end of the Radio Arc (Yusef-Zadeh et al. 1984; LaRosa et al. 2000; Yusef-Zadeh et al. 2004), a region with, supposedly, enhanced density of cosmic ray electrons. Fukuoka et al. (2009) thus proposed that this emission might be induced by cosmic ray electrons.

\section{Origin of the flares-outbursts}

The study of the environment around Sgr A* suggests at least two periods of enhanced activity for the supermassive $\mathrm{BH}$ at the Milky Way centre (see 4.4 ), the first about $6 \pm 2 \times 10^{6} \mathrm{yr}$ ago, with $\mathrm{Sgr} \mathrm{A}^{*}$ emitting close to the Eddington limit, and the second with a luminosity $1-5 \times 10^{39} \mathrm{erg} \mathrm{s}^{-1}$, which occurred $\sim 10^{2} \mathrm{yr}$ ago.

Traces of what triggered the earlier of these accretion events can be found in the close proximity of Sgr A*. The cluster of young stars in the central parsec (see 3.4 suggests the presence of a very massive disc there, $\sim 6 \times 10^{6} \mathrm{yr}$ ago. Some have suggested (although the origin of such disc is still controversial) that this disc 
might have formed from a MC passing very close to Sgr A*. As a result of tidal disruption and stretching, the cloud would follow a range of intersecting orbits, near the circularisation radius, forming an eccentric, clumpy and filamentary disk. The physical conditions in the disk would allow star formation and accretion onto the BH (Sanders 1998; Bonnell \& Rice 2008; Genzel et al. 2010; Yusef-Zadeh \& Wardle 2012). Part of the matter in the inner part of this disc would have been accreted, making Sgr A* shine close to the Eddington limit. Part of the disc would have formed massive stars (the central cluster). The outer part of the disc might have been left as a remnant disc and could now comprise what we call the CND (see 3.3). Finally, a part of the disk would have formed an outflow, such as commonly seen in luminous AGN, perhaps generating both a jet (Su \& Finkbeiner 2012) and inflating the Fermi bubbles (Su et al. 2010).

Somewhat more ambiguous is the physical origin of the enhanced emission that occurred $\sim 10^{2}$ yr ago. Sgr A*'s quiescent X-ray luminosity (see 1.1 is $L_{2-10 \mathrm{keV}}=2 \times 10^{33} \mathrm{erg} \mathrm{s}^{-1}$ (Baganoff et al. 2003) and even during the brightest X-ray flare ever observed (in more than 10 years of XMM-Newton and Chandra monitoring) the X-ray luminosity was only $L_{2-10 \mathrm{keV}}=3 \times 10^{35} \mathrm{erg} \mathrm{s}^{-1}$ (Porquet et al. 2003). The luminosity required to produce the $X$-ray echo seen from the MC, is $L_{2-100 \mathrm{keV}}=1.5-5 \times 10^{39} \mathrm{erg} \mathrm{s}^{-1}$ (Terrier et al. 2010) and is about $\sim 10^{4}$ times higher than the brightest flare ever observed. This seems to suggest an intrinsic difference between the process producing the daily flaring activity and this major flare-outburst. It is rather surprising then that the required X-ray luminosity can be inferred by simply extrapolating the observed K-band flux distribution, obtained over $\sim 10$ years of monitoring, to higher fluxes and rare events (Witzel et al. 2012, Dodds-Eden et al. 2011). This extrapolation to high fluxes and the assumed IR to $\mathrm{X}$-ray conversion involve many assumptions (such as the flare SED and a direct correspondence between the IR and X-ray light curves). Despite these uncertainties, the extrapolation seems to suggest that the X-ray echo is simply an extreme manifestation of the same physical processes that produce the daily flux variability. From a theoretical point of view this conjecture seems plausible. In fact, during normal flux states $\mathrm{Sgr} \mathrm{A}^{*}$ is thought to accrete from the capture of stellar wind material from nearby stars (see 1.1. Cuadra et al. (2008) suggested that, during this process, cold clumps might sometimes fall into the inner region with an angular momentum low enough to circularise at a very small radius $(\sim 0.001$ arcsec $)$, and create there an accretion disc. Such an event might have enhanced the luminosity to $L_{B o l} \sim 10^{40}$ $\mathrm{erg} \mathrm{s}^{-1}$ for a timescale of the order of $\sim 10^{2} \mathrm{yr}$ (dominated by the disc viscous timescale; Cuadra et al. 2008) and have produced the X-ray luminosity required for the observed $\mathrm{Fe} \mathrm{K} \alpha$ echo.

Yu et al. (2011) suggested, instead, that Sgr A*'s flare could be produced by the shock created by the jet deceleration (and not by accretion) caused by a partial capture of a star by the supermassive $\mathrm{BH}$. This model well reproduces the $\mathrm{Fe} \mathrm{K} \alpha$ emission (both the flux and its evolution) of Sgr B2, with a decay time of $\sim 10 \mathrm{yr}$ (Nobukawa et al. 2011; Terrier et al. 2010; Inui et al. 2009; Revnivtsev et al. 2004). Zubovas et al. (2012) consider the consequences of an asteroid passing within a few astronomical units from Sgr A* and being gravitationally disrupted. Objects 
with radius $r \sim 10-50 \mathrm{~km}$ are expected to produce $L_{x} \lesssim 10^{36} \mathrm{erg} \mathrm{s}^{-1}$, thus possibly contributing to Sgr A*'s normal daily flaring activity. However, a planet disruption event (although expected to be much less frequent, e.g. one per $\sim 10^{5} \mathrm{yr}$ ) might produce flares with $L \sim 10^{41} \mathrm{erg} \mathrm{s}^{-1}$ for a few tens of years (Zubovas et al. 2012). Alternatively, Sazonov et al. (2012) considered gas supply onto Sgr A* via tidal interaction of stars. They estimated that every few $10^{4} \mathrm{yr}$, less than $0.1 \mathrm{M}_{\odot}$ of stellar debris will accrete onto Sgr A* and produce an outburst of $L \sim 10^{42-43} \mathrm{erg} \mathrm{s}^{-1}$. A lower luminosity flare could be produced by gas torn off a star that experienced a close collision with another star or a stellar remnant in the nuclear cluster.

Accretion of interstellar material is perhaps a more probable and frequent occurrence because of its larger cross section. In fact, the vicinity of Sgr A* contains several ionised gas streams (Zhao et al. 2009) and numerous blobs of dust and gas (Ghez et al. 2005; Muzić et al. 2010). Thanks to the analysis of more than ten years of infra-red monitoring observations of the stellar orbits of the stars close to Sgr $A^{*}$, it has been recently discovered that a dense cloud (G2) with a mass of at least $\sim 3 \mathrm{M}_{\text {Earth }}$ (estimated from the $\mathrm{Br} \gamma$ luminosity), is now moving (and accelerating) towards Sgr A* (Gillessen et al. 2012a,b). The centre of gravity of the cloud should be at the pericentre (at only 3100 times the BH event horizon) in mid-2013. Due to the interaction between the gas in the cloud and the ambient material, dynamical instabilities (such as Rayleigh-Taylor and Kelvin-Helmholtz instabilities) will disrupt and fragment the cloud into pieces, some of which might accrete onto Sgr A*. It is predicted that the cloud will generate a small increase in the $2-8 \mathrm{keV}$ luminosity of only one order of magnitude above the quiescent level (Burkert et al. 2012; Schartmann et al. 2012). However, the cloud origin, mass content and the effects for the future activity of Sgr A* are still highly debated (Miranda-Escude 2012; Narayan et al. 2012; Anninos et al. 2012). Murray-Clay \& Loeb (2012) suggested that this cloud naturally originates from a proto-planetary disc surrounding an undetected low mass star. If so, the mass accreted onto Sgr A* (and thus Sgr A*'s luminosity) might be orders of magnitude higher producing a rare and bright flare. Further observations of G2 will give us an unprecedented opportunity to study one possible mode of accretion onto a supermassive $\mathrm{BH}$.

\section{An AGN torus in our backyard!}

Several pieces of evidence suggest that $\mathrm{Sgr} \mathrm{A}^{*}$ might have undergone a period of AGN activity about $6 \times 10^{6} \mathrm{yr}$ ago (see 44.4 , Zubovas et al. 2011; 2012). In those conditions the CMZ would have appeared, to an extragalactic observer, to have the properties of a typical AGN torus commonly invoked by AGN unification models (Antonucci 1993; Urry \& Padovani 1995). In fact, it would have obscured (with column densities up to $N_{H} \sim 10^{24-25} \mathrm{~cm}^{-2}$ ) the direct vision of the central BH to an equatorial observer. At about 50-150 pc the height of the CMZ is about several tens of parsec, thus generating obscuration toward a significant fraction of line of sights. This number is not too dissimilar from the one typically estimated from the 
fraction of obscured/unobscured AGN (Urry \& Padovani 1995; Risaliti et al. 1999). The kinematics of the EMR (see $\$ 3.2$ ) is modified by the influence of the stellar bar potential (Binney 1994). However, the radial component of the gas motion could have been generated by the radiation pressure and/or the momentum of an outflow produced by accretion onto $\mathrm{Sgr} \mathrm{A}^{*}$, that might have pushed away the molecular material from the accretion disc axis (Sofue et al. 1995a,b), carving two holes of lower extinction. However, along the Galactic disc, where most of the molecular mass is concentrated, the CMZ represents an almost impassable barrier to Sgr A*'s radiation/outflows. The mass of the CMZ is in fact large enough to impede equatorial outflows from Sgr A*, even if accreting at the Eddington limit $\left(L_{\mathrm{Edd}} \sim 5 \times 10^{44} \mathrm{erg}\right.$ $\mathrm{s}^{-1}$ ). During the last AGN phase, the inner faces of the inner CMZ clouds would have seen the nuclear continuum (efficiently producing UV and X-ray radiation; Elvis et al. 1994) unobscured and be heated by photoionisation, initially to temperatures of $\sim 10^{4} \mathrm{~K}$, then to higher temperatures as their material evaporates away (Krolik \& Begelman 1988). Part of this gas might have generated an outflowing wind (Krolik \& Kriss 1995; 2001), possibly forming a warm absorber component such as observed in UV/X-ray spectra of AGN (Blustin et al. 2005; Kaastra et al. 2012; Kriss et al. 2011), while the remaining matter would have probably been channelled inwards at a rate sufficient to maintain a steady state against evaporation of the torus inner edge (provided that new material can be fed into the outer edge of the torus; Krolik \& Begelman 1988). The luminosity of the nuclear source sets a limit to the survival of dust. Therefore, the so called "dust sublimation radius" is typically assumed to be the inner edge of the molecular torus (Barvainis 1987).

The advent of mid and near-IR interferometric observations at VLT and Keck has recently allowed a dramatic improvement in angular resolution. The application of this technique to a few nearby AGN has allowed investigators to marginally and partially resolve the dust sublimation region (the inner edge of the torus) in these objects (Swain et al. 2003; Jaffe et al. 2004; Tristam et al. 2007; Meisenheimer et al. 2007; Burtscher et al. 2009; Kishimoto et al. 2011a,b). Near-IR observations of AGN (tracing hot, $T \sim 10^{3} \mathrm{~K}$, dust emission) reveal geometrically-thin ring radii on sub-parsec $(\sim 0.03-0.5 \mathrm{pc})$ scales, consistent with the near-IR reverberation radius (the light crossing distance corresponding to the delay between the variabilities in the UV/optical and near-IR; Kishimoto et al. 2011). Moreover, the ring radii present a dependence with source luminosity, as expected for the dust sublimation radii (Kishimoto et al. 2011a,b; Barvainis 1987). Mid-IR observations (tracing warm, $T \sim 10^{2-3} \mathrm{~K}$, dust) display significantly larger radii with a size going from a fraction up to several parsecs. This clearly indicates a stratification in temperature of the radial structure of the torus, with the hot near-IR emission tracing the inner torus edge, closer to the irradiating source. IR observations are thus dominated by a central brightness concentration at the scale of the sublimation radius, the bright rim of the innermost dust distribution, and weaker emission from the external, weakly irradiated, part of the torus (Kishimoto et al. 2011a,b). More difficult is to determine the structure of the outer, weaker emission or any substructure within these AGN tori. 
These results do not contradict the idea that the CND might be the relic of the inner part of the Milky Way AGN torus. Once the intense feeding onto Sgr A* stopped, photo-evaporation halted and the CND has been left as a relic (Duschl 1989) just outside the sublimation radius. The orbital time of the CND material is much shorter than $10^{6} \mathrm{yr}$, so it is unlikely that it kept the same shape that it might have had during the AGN phase. After a long period of quiescence, we do not expect to observe remnant radiation from the emitting regions surrounding a typical AGN, i.e. the broad and narrow line regions, the warm absorber, etc. In fact, the recombination timescales of such plasmas are expected to be much shorter than $\sim 10^{6}$ yr (Osterbrock \& Mathews 1986).

Being only $\sim 8 \mathrm{kpc}$ away (i.e. $\sim 500$ times closer than the closest AGN, Cen A), the GC provides us with the unique opportunity to resolve spatially the internal structure of an AGN torus in such exquisite detail. It is expected that the outer CMZ structure has not changed significantly since the last AGN phase $\left(\sim 6 \times 10^{6} \mathrm{yr}\right.$ ago). In fact, a typical MC core, at $\sim 100 \mathrm{pc}$ from the centre, moving at $\sim 100 \mathrm{~km} \mathrm{~s}^{-1}$ (typical orbital velocities of a MC of the CMZ disc population) would require about $10^{7} \mathrm{yr}$ to complete a full orbit. This suggests that the external structure of the past AGN torus is still preserved in the CMZ. Thus the CMZ can be used as a template to study the structure of the tori of distant AGN and the mass accretion from the outer to inner boundary of AGN tori.

The study of Sgr A*'s surroundings has allowed us to hint at its past activity and the impact of Sgr A*'s emissions on the surrounding matter and star forming regions. Zubovas et al. (2011) estimate that, to power the last AGN phase, Sgr A* might have accreted about $\sim 2 \times 10^{3} \mathrm{M}_{\odot}$. This is about one order of magnitude smaller than the estimated mass stored in the CND. The irregular and clumpy structures of the CND, suggest dynamical evolution and episodic feeding of gas towards Sgr A* (Liu et al. 2012). The CND appears to be the convergence of the innermost parts of large-scale gas streamers, which are responding to the central gravitational potential well. All these indications suggest that the CND might feed the supermassive BH again. Morris et al. (1999) proposed a limit cycle of recurrent nuclear activity where, in the absence of dynamical or radiation pressure from within the CND cavity, viscous disc evolution would cause the inner edge of the CND to shrink, eventually fuelling the BH. The associated burst of radiation would presumably produce shocks and compression of the surrounding gas, possibly inducing a dramatic episode of star formation that could produce a massive cluster like the present central parsec cluster of young stars. The collective winds and radiation pressure from the newly-formed stars and/or AGN accretion, might clear gas from the central region, halting accretion and creating an inner cavity in the CND. The stellar winds from the massive cluster of newborn stars fade out about $\sim 10^{7} \mathrm{yr}$ after the star formation event and, at that point, the inner edge of the CND would start migrate inward again, thus completing the cycle (Morris et al. 1999).

On larger scales, studies of the CMZ structure also suggest that episodic cloud formation and starbursts in the GC region (with a relaxation oscillator mechanism for quasi-periodic starbursts of $\sim 10^{6}-10^{7}$ years; Stark et al. 2004; Martin et al. 2004; Morris \& Serabyn 1996) are a natural result of the interaction between the 
stellar bar and interstellar gas. This star formation cycle, as well as the cloud kinematics and evolution, can be influenced by Sgr A*'s activity, which in turn will be triggered by matter migrating into its sphere of influence. Therefore, the study of Sgr A*'s past activity and its connection to observable phenomena in the CMZ provide a high-resolution opportunity for studying the AGN feedback phenomenon.

Acknowledgements The authors thank the ISSI in Bern. GP thanks S. Bianchi, E. Churazov, G. Matt, S. Gillessen, V. Tatischeff, T. Dwelly, A. Strong, L. Burtscher, A. Merloni, Y. Tanaka and F. Melia for useful discussion. Part of the work was supported by the French Labex "UnivEarthS".

\section{References}

Amo-Baladrón et al.(2009). Amo-Baladrón, M. A., Martín-Pintado, J., Morris, M. R., Muno, M. P., \& Rodríguez-Fernández, N. J. 2009, ApJ, 694, 943

Anninos et al.(2012). Anninos, P., Fragile, P. C., Wilson, J., \& Murray, S. D. 2012, arXiv:1209.1638

Antonucci(1993). Antonucci, R. 1993, ARA\&A, 31, 473

Armstrong \& Barrett(1985). Armstrong, J. T., \& Barrett, A. H. 1985, ApJS, 57, 535

Baganoff et al.(2001). Baganoff, F. K., et al. 2001, Nature, 413, 45

Baganoff et al.(2003). Baganoff, F. K., Maeda, Y., Morris, M., et al. 2003, ApJ, 591, 891

Bally et al.(1987). Bally, J., Stark, A. A., Wilson, R. W., \& Henkel, C. 1987, ApJS, 65, 13

Bally et al.(1988). Bally, J., Stark, A. A., Wilson, R. W., \& Henkel, C. 1988, ApJ, 324, 223

Bally \& Leventhal(1991). Bally, J., \& Leventhal, M. 1991, Nature, 353, 234

Bamba et al.(2002). Bamba, A., Murakami, H., Senda, A., et al. 2002, arXiv:astro-ph/0202010

Bania(1977). Bania, T. M. 1977, ApJ, 216, 381

Barvainis(1987). Barvainis, R. 1987, ApJ, 320, 537

Basko et al.(1974). Basko, M. M., Sunyaev, R. A., \& Titarchuk, L. G. 1974, A\&A, 31, 249

Basko(1978). Basko, M. M. 1978, ApJ, 223, 268

Becklin \& Neugebauer(1968). Becklin, E. E., \& Neugebauer, G. 1968, ApJ, 151, 145

Becklin et al.(1978). Becklin, E. E., Neugebauer, G., Willner, S. P., \& Matthews, K. 1978, ApJ, 220,831

Becklin et al.(1982). Becklin, E. E., Gatley, I., \& Werner, M. W. 1982, ApJ, 258, 135

Belloni \& Hasinger(1990). Belloni, T., \& Hasinger, G. 1990, A\&A, 227, L33

Belloni \& Hasinger(1990). Belloni, T., \& Hasinger, G. 1990, A\&A, 230, 103

Belmont et al.(2005). Belmont, R., Tagger, M., Muno, M., Morris, M., \& Cowley, S. 2005, ApJL, 631, L53

Belmont \& Tagger(2006). Belmont, R., \& Tagger, M. 2006, A\&A, 452, 15

Berezinskii et al.(1990). Berezinskii, V. S., Bulanov, S. V., Dogiel, V. A., \& Ptuskin, V. S. 1990, Amsterdam: North-Holland, 1990, edited by Ginzburg, V.L.,

Bernard et al.(2010). Bernard, J.-P., Paradis, D., Marshall, D. J., et al. 2010, A\&A, 518, L88

Bethe \& Salpeter(1957). Bethe, H. A., \& Salpeter, E. E. 1957, Quantum Mechanics of One- and Two-Electron Atoms, New York: Academic Press, 1957,

Bica et al.(2006). Bica, E., Bonatto, C., Barbuy, B., \& Ortolani, S. 2006, A\&A, 450, 105

Binney et al.(1991). Binney, J., Gerhard, O. E., Stark, A. A., Bally, J., \& Uchida, K. I. 1991, MNRAS, 252, 210

Binney(1994). Binney, J. 1994, NATO ASIC Proc. 445: The Nuclei of Normal Galaxies: Lessons from the Galactic Center, 75

Bland-Hawthorn \& Cohen(2003). Bland-Hawthorn, J., \& Cohen, M. 2003, ApJ, 582, 246

Bleach et al.(1972). Bleach, R. D., Boldt, E. A., Holt, S. S., Schwartz, D. A., \& Serlemitsos, P. J. 1972, ApJL, 174, L101 
Blum et al.(1995). Blum, R. D., Depoy, D. L., \& Sellgren, K. 1995, ApJ, 441, 603

Blustin et al.(2005). Blustin, A. J., Page, M. J., Fuerst, S. V., Branduardi-Raymont, G., \& Ashton, C. E. 2005, A\&A, 431, 111

Bondi(1952). Bondi, H. 1952, MNRAS, 112, 195

Bonnell \& Rice(2008). Bonnell, I. A., \& Rice, W. K. M. 2008, Science, 321, 1060

Bowyer et al.(1965). Bowyer, S., Byram, E. T., Chubb, T. A., \& Friedman, H. 1965, Science, 147, 394

Bradt et al.(1968). Bradt, H., Naranan, S., Rappaport, S., \& Spada, G. 1968, ApJ, 152, 1005

Breitschwerdt et al.(1991). Breitschwerdt, D., McKenzie, J. F., \& Voelk, H. J. 1991, A\&A, 245, 79

Brown \& Gould(1970). Brown, R. L., \& Gould, R. J. 1970, Physical Review D, 1, 2252

Burkert et al.(2012). Burkert, A., Schartmann, M., Alig, C., et al. 2012, ApJ, 750, 58

Bykov(2002). Bykov, A. M. 2002, A\&A, 390, 327

Bykov(2003). Bykov, A. M. 2003, A\&A, 410, L5

Capelli et al.(2011). Capelli, R., Warwick, R. S., Porquet, D., Gillessen, S., \& Predehl, P. 2011, A\&A, 530, A38

Capelli et al.(2012). Capelli, R., Warwick, R. S., Porquet, D., Gillessen, S., \& Predehl, P. 2012, A\&A, 545, A35

Christopher et al.(2005). Christopher, M. H., Scoville, N. Z., Stolovy, S. R., \& Yun, M. S. 2005, ApJ, 622, 346

Churazov et al.(1993). Churazov, E., Gilfanov, M., Sunyaev, R., et al. 1993, ApJ, 407, 752

Churazov et al.(1994). Churazov, E., Gilfanov, M., Sunyaev, R., et al. 1994, ApJS, 92, 381

Churazov et al.(1996). Churazov, E., Gilfanov, M., \& Sunyaev, R. 1996, ApJL, 464, L71

Churazov et al.(2002). Churazov, E., Sunyaev, R., \& Sazonov, S. 2002, MNRAS, 330, 817

Clark et al.(1965). Clark, G., Garmire, G., Oda, M., \& Wada, M. 1965, Nature, 207, 584

Coil et al.(2000). Coil, A. L., et al. 2000, ApJL, 544, L111

Coker \& Melia(1997). Coker, R. F., \& Melia, F. 1997, ApJL, 488, L149

Comastri(2004). Comastri, A. 2004, Supermassive Black Holes in the Distant Universe, 308, 245

Compiègne et al.(2011). Compiègne, M., Verstraete, L., Jones, A., et al. 2011, A\&A, 525, A103

Contopoulos \& Mertzanides(1977). Contopoulos, G., \& Mertzanides, C. 1977, A\&A, 61, 477

Cooke et al.(1970). Cooke, B. A., Griffiths, R. E., \& Pounds, K. A. 1970, Non-Solar X- and Gamma-Ray Astronomy, 37, 280

Coriat et al.(2012). Coriat, M., Fender, R. P., \& Dubus, G. 2012, MNRAS, 424, 1991

Covino et al.(2000). Covino, S., Tagliaferri, G., Pallavicini, R., Mewe, R., \& Poretti, E. 2000, A\&A, 355, 681

Cramphorn \& Sunyaev(2002). Cramphorn, C. K., \& Sunyaev, R. A. 2002, A\&A, 389, 252

Crocker et al.(2010). Crocker, R. M., Jones, D. I., Melia, F., Ott, J., \& Protheroe, R. J. 2010 , Nature, 463, 65

Crocker \& Aharonian(2011). Crocker, R. M., \& Aharonian, F. 2011, Physical Review Letters, 106, 101102

Crocker et al.(2011). Crocker, R. M., Jones, D. I., Aharonian, F., et al. 2011, MNRAS, 413, 763

Cuadra et al.(2005). Cuadra, J., Nayakshin, S., Springel, V., \& Di Matteo, T. 2005, MNRAS, 360, L55

Cuadra et al.(2006). Cuadra, J., Nayakshin, S., Springel, V., \& Di Matteo, T. 2006, MNRAS, 366, 358

Cuadra et al.(2008). Cuadra, J., Nayakshin, S., \& Martins, F. 2008, MNRAS, 383, 458

Dahmen et al.(1998). Dahmen, G., Huttemeister, S., Wilson, T. L., \& Mauersberger, R. 1998, A\&A, 331, 959

Dambis(2009). Dambis, A. K. 2009, MNRAS, 396, 553

Degenaar \& Wijnands(2009). Degenaar, N., \& Wijnands, R. 2009, A\&A, 495, 547

Degenaar \& Wijnands(2010). Degenaar, N., \& Wijnands, R. 2010, A\&A, 524, A69

Degenaar et al.(2012). Degenaar, N., Wijnands, R., Cackett, E. M., et al. 2012, A\&A, 545, A49

de Vaucouleurs(1964). de Vaucouleurs, G. 1964, The Galaxy and the Magellanic Clouds, 20, 195

Dobler \& Finkbeiner(2008). Dobler, G., \& Finkbeiner, D. P. 2008, ApJ, 680, 1222

Dodds-Eden et al.(2011). Dodds-Eden, K., Gillessen, S., Fritz, T. K., et al. 2011, ApJ, 728, 37 
Dogiel et al.(1998). Dogiel, V. A., Ichimura, A., Inoue, H., \& Masai, K. 1998, PASJ, 50, 567

Dogiel et al.(2002). Dogiel, V. A., Inoue, H., Masai, K., Schönfelder, V., \& Strong, A. W. 2002, ApJ, 581, 1061

Dogiel et al.(2009). Dogiel, V., et al. 2009, PASJ, 61, 901

Dogiel et al.(2011). Dogiel, V., Chernyshov, D., Koyama, K., Nobukawa, M., \& Cheng, K.-S. 2011, PASJ, 63, 535

Duschl(1989). Duschl, W. J. 1989, MNRAS, 240, 219

Dwek et al.(1995). Dwek, E., Arendt, R. G., Hauser, M. G., et al. 1995, ApJ, 445, 716

Eisenhauer et al.(2003). Eisenhauer, F., Schödel, R., Genzel, R., Ott, T., Tecza, M., Abuter, R., Eckart, A., \& Alexander, T. 2003, ApJL, 597, L121

Elvis et al.(1994). Elvis, M., Wilkes, B. J., McDowell, J. C., et al. 1994, ApJS, 95, 1

Fabian(1977). Fabian, A. C. 1977, Nature, 269, 672

Favata et al.(1997). Favata, F., Micela, G., \& Sciortino, S. 1997, A\&A, 326, 647

Fender et al.(2004). Fender, R. P., Belloni, T. M., \& Gallo, E. 2004, MNRAS, 355, 1105

Fender \& Belloni(2004). Fender, R., \& Belloni, T. 2004, ARA\&A, 42, 317

Ferrière(2009). Ferrière, K. 2009, A\&A, 505, 1183

Figer et al.(2002). Figer, D. F., Najarro, F., Gilmore, D., et al. 2002, ApJ, 581, 258

Fisher et al.(1966). Fisher, P. C., Johnson, H. M., Jordan, W. C., Meyerott, A. J., \& Acton, L. W. 1966, ApJ, 143, 203

Fritz et al.(2011). Fritz, T. K., Gillessen, S., Dodds-Eden, K., et al. 2011, ApJ, 737, 73

Fryer et al.(2006). Fryer, C. L., Rockefeller, G., Hungerford, A., \& Melia, F. 2006, ApJ, 638, 786

Fukuoka et al.(2009). Fukuoka, R., Koyama, K., Ryu, S. G., \& Tsuru, T. G. 2009, PASJ, 61, 593

Garcia et al.(1973). Garcia, J. D., Fortner, R. J., \& Kavanagh, T. M. 1973, Reviews of Modern Physics, 45,111

Genzel et al.(1985). Genzel, R., Crawford, M. K., Townes, C. H., \& Watson, D. M. 1985, ApJ, 297, 766

Genzel et al.(1996). Genzel, R., Thatte, N., Krabbe, A., Kroker, H., \& Tacconi-Garman, L. E. 1996, ApJ, 472, 153

Genzel et al.(2000). Genzel, R., Pichon, C., Eckart, A., Gerhard, O. E., \& Ott, T. 2000, MNRAS, 317,348

Genzel et al.(2003). Genzel, R., Schödel, R., Ott, T., et al. 2003, ApJ, 594, 812

Genzel et al.(2003). Genzel, R., Schödel, R., Ott, T., Eckart, A., Alexander, T., Lacombe, F., Rouan, D., \& Aschenbach, B. 2003, Nature, 425, 934

Genzel et al.(2010). Genzel, R., Eisenhauer, F., \& Gillessen, S. 2010, Reviews of Modern Physics, 82,3121

George \& Fabian(1991). George, I. M., \& Fabian, A. C. 1991, MNRAS, 249, 352

Ghez et al.(2003). Ghez, A. M., Duchêne, G., Matthews, K., et al. 2003, ApJL, 586, L127

Ghez et al.(2003). Ghez, A. M., Becklin, E., Duchjne, G., Hornstein, S., Morris, M., Salim, S., \& Tanner, A. 2003, Astronomische Nachrichten Supplement, 324, 527

Ghez et al.(2004). Ghez, A. M., et al. 2004, ApJL, 601, L159

Ghez et al.(2005). Ghez, A. M., et al. 2005, ApJ, 635, 1087

Ghez et al.(2008). Ghez, A. M., Salim, S., Weinberg, N. N., et al. 2008, ApJ, 689, 1044

Ghisellini et al.(1994). Ghisellini, G., Haardt, F., \& Matt, G. 1994, MNRAS, 267, 743

Gillessen et al.(2009). Gillessen, S., Eisenhauer, F., Fritz, T. K., et al. 2009, ApJL, 707, L114

Gillessen et al.(2009). Gillessen, S., Eisenhauer, F., Trippe, S., Alexander, T., Genzel, R., Martins, F., \& Ott, T. 2009, ApJ, 692, 1075

Gillessen et al.(2012). Gillessen, S., Genzel, R., Fritz, T. K., et al. 2012, Nature, 481, 51

Gillessen et al.(2012). Gillessen, S., Genzel, R., Fritz, T. K., et al. 2012, arXiv:1209.2272

Glass et al.(1987). Glass, I. S., Catchpole, R. M., \& Whitelock, P. A. 1987, MNRAS, 227, 373

Goldwurm et al.(1994). Goldwurm, A., Cordier, B., Paul, J., et al. 1994, Nature, 371, 589

Goldwurm(2001). Goldwurm, A. 2001, Exploring the Gamma-Ray Universe, 459, 455

Goldwurm et al.(2003). Goldwurm, A., Brion, E., Goldoni, P., Ferrando, P., Daigne, F., Decourchelle, A., Warwick, R. S., \& Predehl, P. 2003, ApJ, 584, 751

Groenewegen et al.(2008). Groenewegen, M. A. T., Udalski, A., \& Bono, G. 2008, A\&A, 481, 441 
Gualandris et al.(2012). Gualandris, A., Mapelli, M., \& Perets, H. B. 2012, arXiv:1209.3021

Gursky et al.(1967). Gursky, H., Gorenstein, P., \& Giacconi, R. 1967, ApJL, 150, L75

Guesten \& Downes(1980). Guesten, R., \& Downes, D. 1980, A\&A, 87, 6

Guesten et al.(1981). Guesten, R., Walmsley, C. M., \& Pauls, T. 1981, A\&A, 103, 197

Guesten et al.(1987). Guesten, R., Genzel, R., Wright, M. C. H., et al. 1987, ApJ, 318, 124

Güsten(1989). Güsten, R. 1989, The Center of the Galaxy, 136, 89

Handa et al.(2006). Handa, T., Sakano, M., Naito, S., Hiramatsu, M., \& Tsuboi, M. 2006, ApJ, 636,261

Harfst et al.(2010). Harfst, S., Portegies Zwart, S., \& Stolte, A. 2010, MNRAS, 409, 628

Heiligman(1987). Heiligman, G. M. 1987, ApJ, 314, 747

Hobbs \& Nayakshin(2009). Hobbs, A., \& Nayakshin, S. 2009, MNRAS, 394, 191

in't Zand et al.(2004). in't Zand, J., Verbunt, F., Heise, J., et al. 2004, Nuclear Physics B Proceedings Supplements, 132, 486

Inui et al.(2009). Inui, T., Koyama, K., Matsumoto, H., \& Tsuru, T. G. 2009, PASJ, 61, 241

Jackson et al.(1993). Jackson, J. M., Geis, N., Genzel, R., et al. 1993, ApJ, 402, 173

Jackson et al.(1996). Jackson, J. M., Heyer, M. H., Paglione, T. A. D., \& Bolatto, A. D. 1996, ApJL, 456, L91

Kaastra et al.(2012). Kaastra, J. S., Detmers, R. G., Mehdipour, M., et al. 2012, A\&A, 539, A117

Kaifu et al.(1972). Kaifu, N., Kato, T., \& Iguchi, T. 1972, Nature Physical Science, 238, 105

Kaneda et al.(1997). Kaneda, H., Makishima, K., Yamauchi, S., et al. 1997, ApJ, 491, 638

Kennea et al.(2006). Kennea, J. A., Burrows, D. N., Campana, S., et al. 2006, The Astronomer's Telegram, 753, 1

Kimura et al.(2010). Kimura, M., Maihara, T., Iwamuro, F., et al. 2010, PASJ, 62, 1135

Körding et al.(2007). Körding, E. G., Migliari, S., Fender, R., et al. 2007, MNRAS, 380, 301

Koyama et al.(1986). Koyama, K., Makishima, K., Tanaka, Y., \& Tsunemi, H. 1986, PASJ, 38, 121

Koyama et al.(1989). Koyama, K., Awaki, H., Kunieda, H., Takano, S., \& Tawara, Y. 1989, Nature, 339,603

Koyama et al.(1996). Koyama, K., Maeda, Y., Sonobe, T., et al. 1996, PASJ, 48, 249

Koyama et al.(2007). Koyama, K., Inui, T., Hyodo, Y., et al. 2007, PASJ, 59, 221

Koyama et al.(2007). Koyama, K., et al. 2007, PASJ, 59, 245

Koyama et al.(2008). Koyama, K., Inui, T., Matsumoto, H., \& Tsuru, T. G. 2008, PASJ, 60, 201

Koyama et al.(2009). Koyama, K., Takikawa, Y., Hyodo, Y., Inui, T., Nobukawa, M., Matsumoto, H., \& Tsuru, T. G. 2009, PASJ, 61, 255

Koyama(2011). Koyama, K. 2011, The Galactic Center: a Window to the Nuclear Environment of Disk Galaxies, 439, 418

Kriss et al.(2011). Kriss, G. A., Arav, N., Kaastra, J. S., et al. 2011, A\&A, 534, A41

Krolik \& Begelman(1988). Krolik, J. H., \& Begelman, M. C. 1988, ApJ, 329, 702

Krolik et al.(1994). Krolik, J. H., Madau, P., \& Zycki, P. T. 1994, ApJL, 420, L57

Krolik \& Kriss(1995). Krolik, J. H., \& Kriss, G. A. 1995, ApJ, 447, 512

Krolik \& Kriss(2001). Krolik, J. H., \& Kriss, G. A. 2001, ApJ, 561, 684

Kuntz \& Snowden(2008). Kuntz, K. D., \& Snowden, S. L. 2008, ApJ, 674, 209

Kuulkers et al.(2007). Kuulkers, E., Shaw, S. E., Paizis, A., et al. 2007, A\&A, 466, 595

in't Zand et al.(2004). in't Zand, J., Verbunt, F., Heise, J., et al. 2004, Nuclear Physics B Proceedings Supplements, 132, 486

Irvine et al.(1987). Irvine, W. M., Goldsmith, P. F., \& Hjalmarson, A. 1987, Interstellar Processes, 134,561

LaRosa et al.(2000). LaRosa, T. N., Kassim, N. E., Lazio, T. J. W., \& Hyman, S. D. 2000, AJ, 119,207

LaRosa et al.(2005). LaRosa, T. N., Brogan, C. L., Shore, S. N., et al. 2005, ApJL, 626, L23

Li et al.(2012). Li, Z., Morris, R. M., \& Baganoff, M., 2012, IAU Symposium, Feeding Compact Objects on All Scales, Beijing, China

Liszt \& Burton(1978). Liszt, H. S., \& Burton, W. B. 1978, ApJ, 226, 790

Liszt \& Burton(1980). Liszt, H. S., \& Burton, W. B. 1980, ApJ, 236, 779

Liu et al.(2012). Liu, H. B., Hsieh, P.-Y., Ho, P. T. P., et al. 2012, ApJ, 756, 195 
Lu et al.(2009). Lu, J. R., Ghez, A. M., Hornstein, S. D., et al. 2009, ApJ, 690, 1463

Maeda et al.(2002). Maeda, Y., Baganoff, F. K., Feigelson, E. D., et al. 2002, ApJ, 570, 671

Martin(1970). Martin, P. G. 1970, MNRAS, 149, 221

Martín et al.(2012). Martín, S., Martín-Pintado, J., Montero-Castaño, M., Ho, P. T. P., \& Blundell, R. 2012, A\&A, 539, A29

Martín-Pintado et al.(2000). Martín-Pintado, J., de Vicente, P., Rodríguez-Fernández, N. J., Fuente, A., \& Planesas, P. 2000, A\&A, 356, L5

Masui et al.(2009). Masui, K., Mitsuda, K., Yamasaki, N. Y., et al. 2009, PASJ, 61, 115

Matsunaga et al.(2009). Matsunaga, N., Kawadu, T., Nishiyama, S., et al. 2009, MNRAS, 399, 1709

Matt et al.(1991). Matt, G., Perola, G. C., \& Piro, L. 1991, A\&A, 247, 25

Matt et al.(1996). Matt, G., Brandt, W. N., \& Fabian, A. C. 1996, MNRAS, 280, 823

Matt et al.(1997). Matt, G., Fabian, A. C., \& Reynolds, C. S. 1997, MNRAS, 289, 175

Matt(2002). Matt, G. 2002, MNRAS, 337, 147

Matt et al.(2003). Matt, G., Guainazzi, M., \& Maiolino, R. 2003, MNRAS, 342, 422

Mauerhan et al.(2009). Mauerhan, J. C., Muno, M. P., Morris, M. R., et al. 2009, ApJ, 703, 30

Mauersberger et al.(1986). Mauersberger, R., Henkel, C., Wilson, T. L., \& Walmsley, C. M. 1986, A\&A, 162, 199

McHardy et al.(2006). McHardy, I. M., Koerding, E., Knigge, C., Uttley, P., \& Fender, R. P. 2006, Nature, 444, 730

Melia(1992). Melia, F. 1992, ApJL, 387, L25

Melia \& Falcke(2001). Melia, F., \& Falcke, H. 2001, ARA\&A, 39, 309

Melia(2007). Melia, F. 2007, The Galactic Supermassive Black Hole by Fulvio Melia. Princeton University Press, 2007.,

Miralda-Escudé(2012). Miralda-Escudé, J. 2012, ApJ, 756, 86

Molendi et al.(2003). Molendi, S., Bianchi, S., \& Matt, G. 2003, MNRAS, 343, L1

Molinari et al.(2011). Molinari, S., Bally, J., Noriega-Crespo, A., et al. 2011, ApJL, 735, L33

Montero-Castaño et al.(2009). Montero-Castaño, M., Herrnstein, R. M., \& Ho, P. T. P. 2009, ApJ, 695,1477

Morris et al.(1983). Morris, M., Polish, N., Zuckerman, B., \& Kaifu, N. 1983, AJ, 88, 1228

Morris(1990). Morris, M. 1990, Galactic and Intergalactic Magnetic Fields, 140, 361

Morris et al.(1992). Morris, M., Davidson, J. A., Werner, M., et al. 1992, ApJL, 399, L63

Morris(1996). Morris, M. 1996, Unsolved Problems of the Milky Way, 169, 247

Morris \& Serabyn(1996). Morris, M., \& Serabyn, E. 1996, ARA\&A, 34, 645

Morris et al.(1999). Morris, M., Ghez, A. M., \& Becklin, E. E. 1999, Advances in Space Research, 23, 959

Muno et al.(2003). Muno, M. P., Baganoff, F. K., Bautz, M. W., et al. 2003, ApJ, 589, 225

Muno et al.(2004). Muno, M. P., et al. 2004, ApJ, 613, 1179

Muno et al.(2006). Muno, M. P., Bauer, F. E., Bandyopadhyay, R. M., \& Wang, Q. D. 2006, ApJS, 165,173

Muno et al.(2007). Muno, M. P., Baganoff, F. K., Brandt, W. N., Park, S., \& Morris, M. R. 2007, ApJL, 656, L69

Muno et al.(2009). Muno, M. P., Bauer, F. E., Baganoff, F. K., et al. 2009, ApJS, 181, 110

Murakami et al.(2000). Murakami, H., Koyama, K., Sakano, M., Tsujimoto, M., \& Maeda, Y. 2000, ApJ, 534, 283

Murakami et al.(2001). Murakami, H., Koyama, K., Tsujimoto, M., Maeda, Y., \& Sakano, M. 2001a, ApJ, 550, 297

Murakami et al.(2001). Murakami, H., Koyama, K., \& Maeda, Y. 2001b, ApJ, 558, 687

Murray-Clay \& Loeb(2011). Murray-Clay, R. A., \& Loeb, A. 2011, arXiv:1112.4822

Nakajima et al.(2009). Nakajima, H., Tsuru, T. G., Nobukawa, M., et al. 2009, PASJ, 61, 233

Nandra \& George(1994). Nandra, K., \& George, I. M. 1994, MNRAS, 267, 974

Narayan et al.(2012). Narayan, R., Özel, F., \& Sironi, L. 2012, ApJL, 757, L20

Nayakshin et al.(2007). Nayakshin, S., Cuadra, J., \& Springel, V. 2007, MNRAS, 379, 21

Nishiyama et al.(2006). Nishiyama, S., Nagata, T., Kusakabe, N., et al. 2006, ApJ, 638, 839

Nobukawa et al.(2008). Nobukawa, M., Tsuru, T. G., Takikawa, Y., et al. 2008, PASJ, 60, 191 
Nobukawa et al.(2009). Nobukawa, M., Sawada, M., Matsumoto, H., Tsuru, T. G., \& Koyama, K. 2009, Advances in Space Research, 43, 1045

Nobukawa et al.(2011). Nobukawa, M., Ryu, S. G., Tsuru, T. G., \& Koyama, K. 2011, ApJL, 739, L52

Nottingham et al.(1993). Nottingham, M. R., Skinner, G. K., Willmore, A. P., et al. 1993, Astronomy and Astrophysics, Supplement, 97, 165

Odaka et al.(2011). Odaka, H., Aharonian, F., Watanabe, S., et al. 2011, ApJ, 740, 103

Oka et al.(2005). Oka, T., Geballe, T. R., Goto, M., Usuda, T., \& McCall, B. J. 2005, ApJ, 632, 882

Oka et al.(2011). Oka, T., Nagai, M., Kamegai, K., \& Tanaka, K. 2011, ApJ, 732, 120

Osterbrock \& Mathews(1986). Osterbrock, D. E., \& Mathews, W. G. 1986, ARA\&A, 24, 171

Overbeck(1965). Overbeck, J. W. 1965, ApJ, 141, 864

Palmeri et al.(2003). Palmeri, P., Mendoza, C., Kallman, T. R., Bautista, M. A., \& Meléndez, M. 2003, A\&A, 410, 359

Palmeri et al.(2003). Palmeri, P., Mendoza, C., Kallman, T. R., \& Bautista, M. A. 2003, A\&A, 403,1175

Park et al.(2004). Park, S., Muno, M. P., Baganoff, F. K., et al. 2004, ApJ, 603, 548

Paumard et al.(2006). Paumard, T., Genzel, R., Martins, F., et al. 2006, ApJ, 643, 1011

Pavlinsky et al.(1994). Pavlinsky, M. N., Grebenev, S. A., \& Sunyaev, R. A. 1994, ApJ, 425, 110

Peters(1975). Peters, W. L., III 1975, ApJ, 195, 617

Planck Collaboration et al.(2012). Planck Collaboration, Ade, P. A. R., Aghanim, N., et al. 2012, arXiv: 1208.5483

Ponti et al.(2010). Ponti, G., Terrier, R., Goldwurm, A., Belanger, G., \& Trap, G. 2010, ApJ, 714, 732

Ponti et al.(2011). Ponti, G., Terrier, R., Goldwurm, A., Belanger, G., \& Trap, G. 2011, The Galactic Center: a Window to the Nuclear Environment of Disk Galaxies, 439, 446

Ponti et al.(2012). Ponti, G., Fender, R. P., Begelman, M. C., et al. 2012, MNRAS, 422, L11

Ponti et al.(2012). Ponti, G., Papadakis, I., Bianchi, S., et al. 2012, A\&A, 542, A83

Porquet et al.(2003). Porquet, D., Predehl, P., Aschenbach, B., Grosso, N., Goldwurm, A., Goldoni, P., Warwick, R. S., \& Decourchelle, A. 2003, A\&A, 407, L17

Predehl \& Schmitt(1995). Predehl, P., \& Schmitt, J. H. M. M. 1995, A\&A, 293, 889

Quataert(2004). Quataert, E. 2004, ApJ, 613, 322

Quinn \& Sussman(1985). Quinn, P. J., \& Sussman, G. J. 1985, ApJ, 288, 377

Reid et al.(2009). Reid, M. J., Menten, K. M., Zheng, X. W., et al. 2009, ApJ, 700, 137

Reid et al.(2009). Reid, M. J., Menten, K. M., Zheng, X. W., Brunthaler, A., \& Xu, Y. 2009, ApJ, 705,1548

Requena-Torres et al.(2012). Requena-Torres, M. A., Güsten, R., Weiß, A., et al. 2012, A\&A, 542, L21

Revnivtsev et al.(2003). Revnivtsev, M., Gilfanov, M., Sunyaev, R., Jahoda, K., \& Markwardt, C. 2003, A\&A, 411, 329

Revnivtsev et al.(2004). Revnivtsev, M. G., et al. 2004, A\&A, 425, L49

Revnivtsev et al.(2006). Revnivtsev, M., Sazonov, S., Gilfanov, M., Churazov, E., \& Sunyaev, R. 2006a, A\&A, 452, 169

Revnivtsev et al.(2006). Revnivtsev, M., Molkov, S., \& Sazonov, S. 2006b, MNRAS, 373, L11

Revnivtsev et al. 2007. Revnivtsev, M. G., et al., 2007, $A \& A, 473,857$

Revnivtsev et al.(2009). Revnivtsev, M., Sazonov, S., Churazov, E., et al. 2009, Nature, 458, 1142

Rieke et al.(1989). Rieke, G. H., Rieke, M. J., \& Paul, A. E. 1989, ApJ, 336, 752

Risaliti et al.(1999). Risaliti, G., Maiolino, R., \& Salvati, M. 1999, ApJ, 522, 157

Rockefeller et al.(2004). Rockefeller, G., Fryer, C. L., Melia, F., \& Warren, M. S. 2004, ApJ, 604, 662

Rockefeller et al.(2005). Rockefeller, G., Fryer, C. L., Baganoff, F. K., \& Melia, F. 2005, ApJL, 635, L141

Rodríguez-Fernández et al.(2004). Rodríguez-Fernández, N. J., Martín-Pintado, J., Fuente, A., \& Wilson, T. L. 2004, A\&A, 427, 217 
Rolf(1983). Rolf, D. P. 1983, Nature, 302, 46

Ryu et al.(2009). Ryu, S. G., Koyama, K., Nobukawa, M., Fukuoka, R., \& Tsuru, T. G. 2009, PASJ, 61, 751

Saito(1990). Saito, M. 1990, PASJ, 42, 19

Sakano et al.(2002). Sakano, M., Koyama, K., Murakami, H., Maeda, Y., \& Yamauchi, S. 2002, ApJS, 138, 19

Sakano et al.(2004). Sakano, M., Warwick, R. S., Decourchelle, A., \& Predehl, P. 2004, MNRAS, 350,129

Sanders(1989). Sanders, R. H. 1989, The Center of the Galaxy, 136, 77

Sanders(1998). Sanders, R. H. 1998, MNRAS, 294, 35

Sawada et al.(2004). Sawada, T., Hasegawa, T., Handa, T., \& Cohen, R. J. 2004, MNRAS, 349, 1167

Sazonov et al.(2006). Sazonov, S., Revnivtsev, M., Gilfanov, M., Churazov, E., \& Sunyaev, R. 2006, A\&A, 450, 117

Sazonov et al.(2012). Sazonov, S., Sunyaev, R., \& Revnivtsev, M. 2012, MNRAS, 420, 388

Schödel et al.(2002). Schödel, R., et al. 2002, Nature, 419, 694

Scoville(1972). Scoville, N. Z. 1972, ApJL, 175, L127

Sellwood \& Wilkinson(1993). Sellwood, J. A., \& Wilkinson, A. 1993, Reports on Progress in Physics, 56, 173

Sellwood(1993). Sellwood, J. A. 1993, Back to the Galaxy, 278, 133

Serabyn \& Guesten(1987). Serabyn, E., \& Guesten, R. 1987, A\&A, 184, 133

Schartmann et al.(2012). Schartmann, M., Burkert, A., Alig, C., et al. 2012, ApJ, 755, 155

Schödel et al.(2002). Schödel, R., Ott, T., Genzel, R., et al. 2002, Nature, 419, 694

Schödel et al.(2007). Schödel, R., Eckart, A., Alexander, T., et al. 2007, A\&A, 469, 125

Schödel et al.(2009). Schödel, R., Merritt, D., \& Eckart, A. 2009, A\&A, 502, 91

Sidoli et al.(1999). Sidoli, L., Mereghetti, S., Israel, G. L., et al. 1999, ApJ, 525, 215

Sidoli et al.(2001). Sidoli, L., Belloni, T., \& Mereghetti, S. 2001, A\&A, 368, 835

Sidoli et al.(2001). Sidoli, L., Mereghetti, S., Treves, A., et al. 2001, A\&A, 372, 651

Skinner et al.(1987). Skinner, G. K., Willmore, A. P., Eyles, C. J., Bertram, D., \& Church, M. J. 1987, Nature, 330, 544

Sofue(1995). Sofue, Y. 1995, PASJ, 47, 527

Sofue(1995). Sofue, Y. 1995, PASJ, 47, 551

Stark \& Bania(1986). Stark, A. A., \& Bania, T. M. 1986, ApJL, 306, L17

Stark \& Brand(1989). Stark, A. A., \& Brand, J. 1989, ApJ, 339, 763

Su et al.(2010). Su, M., Slatyer, T. R., \& Finkbeiner, D. P. 2010, ApJ, 724, 1044

Su \& Finkbeiner(2012). Su, M., \& Finkbeiner, D. P. 2012, ApJ, 753, 61

Sunyaev et al.(1991). Sunyaev, R., Churazov, E., Gilfanov, M., et al. 1991, A\&A, 247, L29

Sunyaev et al.(1993). Sunyaev, R. A., Markevitch, M., \& Pavlinsky, M. 1993, ApJ, 407, 606

Sunyaev \& Churazov(1996). Sunyaev, R. A., \& Churazov, E. M. 1996, Astronomy Letters, 22, 648

Sunyaev \& Churazov(1998). Sunyaev, R., \& Churazov, E. 1998, MNRAS, 297, 1279

Syer \& Ulmer(1999). Syer, D., \& Ulmer, A. 1999, MNRAS, 306, 35

Swank \& Markwardt(2001). Swank, J., \& Markwardt, C. 2001, New Century of X-ray Astronomy, 251, 94

Tanaka(2002). Tanaka, Y. 2002, A\&A, 382, 1052

Tanaka \& Yamauchi (2011). anaka, Y. \& Yamauchi 2011, JAXA-SP-09-008E, ISSI 1349-113X

Tanner et al.(2006). Tanner, A., Figer, D. F., Najarro, F., et al. 2006, ApJ, 641, 891

Tatischeff et al.(1998). Tatischeff, V., Ramaty, R., \& Kozlovsky, B. 1998, ApJ, 504, 874

Tatischeff et al.(2003). Tatischeff, V., Duprat, J., Kiener, J., et al. 2003, Physical Review C, 68, 025804

Tatischeff et al.(2012). Tatischeff, V., Decourchelle, A., Maurin, G., 2012, A\&A, in press.

Terrier et al.(2010). Terrier, R., Ponti, G., Bélanger, G., et al. 2010, ApJ, 719, 143

Toor et al.(1976). Toor, A., Palmieri, T. M., \& Seward, F. D. 1976, ApJ, 207, 96

Trippe et al.(2008). Trippe, S., Gillessen, S., Gerhard, O. E., et al. 2008, A\&A, 492, 419 
Tsuboi et al.(1989). Tsuboi, M., Handa, T., Inoue, M., Inatani, J., \& Ukita, N. 1989, The Center of the Galaxy, 136, 135

Tsuboi et al.(1999). Tsuboi, M., Handa, T., \& Ukita, N. 1999, ApJS, 120, 1

Tsujimoto et al.(2007). Tsujimoto, M., Hyodo, Y., \& Koyama, K. 2007, PASJ, 59, 229

Uchiyama et al.(2011). Uchiyama, H., Nobukawa, M., Tsuru, T., Koyama, K., \& Matsumoto, H. 2011, PASJ, 63, 903

Urry \& Padovani(1995). Urry, C. M., \& Padovani, P. 1995, Publications of the ASP, 107, 803

Valinia \& Marshall(1998). Valinia, A., \& Marshall, F. E. 1998, ApJ, 505, 134

Valinia et al.(2000). Valinia, A., Tatischeff, V., Arnaud, K., Ebisawa, K., \& Ramaty, R. 2000, ApJ, 543,733

Vanhollebeke et al.(2009). Vanhollebeke, E., Groenewegen, M. A. T., \& Girardi, L. 2009, A\&A, 498, 95

Vollmer \& Duschl(2002). Vollmer, B., \& Duschl, W. J. 2002, A\&A, 388, 128

Walmsley et al.(1986). Walmsley, C. M., Baudry, A., Guilloteau, S., \& Winnberg, A. 1986, A\&A, 167,151

Warwick et al.(1985). Warwick, R. S., Turner, M. J. L., Watson, M. G., \& Willingale, R. 1985, Nature, 317, 218

Warwick et al.(1988). Warwick, R. S., Norton, A. J., Turner, M. J. L., Watson, M. G., \& Willingale, R. 1988, MNRAS, 232, 551

Wang et al.(2002). Wang, Q. D., Gotthelf, E. V., \& Lang, C. C. 2002, Nature, 415, 148

Wang et al.(2006). Wang, Q. D., Dong, H., \& Lang, C. 2006, MNRAS, 371, 38

Watson et al.(1981). Watson, M. G., Willingale, R., Hertz, P., \& Grindlay, J. E. 1981, ApJ, 250, 142

Witzel et al.(2012). Witzel, G., Eckart, A., Bremer, M., et al. 2012, arXiv:1208.5836

Wijnands et al.(2006). Wijnands, R., in't Zand, J. J. M., Rupen, M., et al. 2006, A\&A, 449, 1117

Xiang et al.(2007). Xiang, J., Lee, J. C., \& Nowak, M. A. 2007, ApJ, 660, 1309

Xiang et al.(2011). Xiang, J., Lee, J. C., Nowak, M. A., \& Wilms, J. 2011, ApJ, 738, 78

Xu et al.(2006). Xu, Y.-D., Narayan, R., Quataert, E., Yuan, F., \& Baganoff, F. K. 2006, ApJ, 640, 319

Yaqoob et al.(2010). Yaqoob, T., Murphy, K. D., Miller, L., \& Turner, T. J. 2010, MNRAS, 401, 411

Yaqoob \& Murphy(2011). Yaqoob, T., \& Murphy, K. D. 2011, MNRAS, 412, 277

Yamauchi et al.(1990). Yamauchi, S., Kawada, M., Koyama, K., Kunieda, H., \& Tawara, Y. 1990, ApJ, 365, 532

Yamauchi \& Koyama(1993). Yamauchi, S., \& Koyama, K. 1993, ApJ, 404, 620

Yamauchi et al.(1996). Yamauchi, S., Kaneda, H., Koyama, K., et al. 1996, PASJ, 48, L15

Yamauchi et al.(2008). Yamauchi, S., Ueno, M., Koyama, K., \& Bamba, A. 2008, PASJ, 60, 1143

Yamauchi et al.(2009). Yamauchi, S., Ebisawa, K., Tanaka, Y., et al. 2009, PASJ, 61, 225

Yoshino et al.(2009). Yoshino, T., Mitsuda, K., Yamasaki, N. Y., et al. 2009, PASJ, 61, 805

Yu et al.(2011). Yu, Y.-W., Cheng, K. S., Chernyshov, D. O., \& Dogiel, V. A. 2011, MNRAS, 411, 2002

Yuasa et al.(2012). Yuasa, T., Makishima, K., \& Nakazawa, K. 2012, ApJ, 753, 129

Yusef-Zadeh et al.(1984). Yusef-Zadeh, F., Morris, M., \& Chance, D. 1984, Nature, 310, 557

Yusef-Zadeh et al.(2002). Yusef-Zadeh, F., Law, C., Wardle, M., Wang, Q. D., Fruscione, A., Lang, C. C., \& Cotera, A. 2002, ApJ, 570, 665

Yusef-Zadeh et al.(2002). Yusef-Zadeh, F., Law, C., \& Wardle, M. 2002, ApJL, 568, L121

Yusef-Zadeh et al.(2004). Yusef-Zadeh, F., Hewitt, J. W., \& Cotton, W. 2004, ApJS, 155, 421

Yusef-Zadeh et al.(2007). Yusef-Zadeh, F., Muno, M., Wardle, M., \& Lis, D. C. 2007, ApJ, 656, 847

Yusef-Zadeh et al.(2012). Yusef-Zadeh, F., Arendt, R., Bushouse, H., et al. 2012, arXiv:1208.1193

Yusef-Zadeh et al.(2012). Yusef-Zadeh, F., Hewitt, J. W., Wardle, M., et al. 2012, arXiv: 1206.6882

Yusef-Zadeh \& Wardle(2012). Yusef-Zadeh, F., \& Wardle, M. 2012, Journal of Physics Conference Series, 372, 012024 
Zhao(1994). Zhao, H. 1994, Ph.D. Thesis,

Zhao et al.(1995). Zhao, J.-H., Goss, W. M., \& Ho, P. T. P. 1995, ApJ, 450, 122

Zeldovich \& Raizer(1966). Zeldovich, Y. B., \& Raizer, Y. P. 1966, New York: Academic Press, 1966, edited by Hayes, W.D.; Probstein, Ronald F.,

Zubovas et al.(2011). Zubovas, K., King, A. R., \& Nayakshin, S. 2011, MNRAS, 415, L21

Zubovas \& Nayakshin(2012). Zubovas, K., \& Nayakshin, S. 2012, MNRAS, 424, 666

Zubovas et al.(2012). Zubovas, K., Nayakshin, S., \& Markoff, S. 2012, MNRAS, 421, 1315 